\title{
Theory of Optimal Orthonormal Subband Coders
}

\author{
P. P. Vaidyanathan, Fellow, IEEE
}

\begin{abstract}
The theory of the orthogonal transform coder and methods for its optimal design have been known for a long time. In this paper, we derive a set of necessary and sufficient conditions for the coding-gain optimality of an orthonormal subband coder for given input statistics. We also show how these conditions can be satisfied by the construction of a sequence of optimal compaction filters one at a time. Several theoretical properties of optimal compaction filters and optimal subband coders are then derived, especially pertaining to behavior as the number of subbands increases. Significant theoretical differences between optimum subband coders, transform coders, and predictive coders are summarized. Finally, conditions are presented under which optimal orthonormal subband coders yield as much coding gain as biorthogonal ones for a fixed number of subbands.
\end{abstract}

Index Terms - Coding gain, optimal filter banks, orthonormal coders, paraunitary coders, principal-component filter banks, subband coders, transform coders.

\section{INTRODUCTION}

$\mathbf{T}$ HE THEORY of the orthogonal transform coder and methods for its optimal design have been known for a long time [1], [2]. Theoretical properties of the optimal solution are well understood [3], and useful practical approximations are routinely employed in data compression today [3]-[6]. The subband coder, which is a generalization of the transform coder, has also been well-known and used in several applications including data compression [3]-[11]. The optimization of subband coders has also been considered to some extent [12]-[22]. Similar to classical transform coders, the optimality is in the sense of maximizing the coding gain, i.e., minimizing the mean square reconstruction error due to subband quantization. In this paper, we will present the general theory of optimal orthonormal subband coders.

Our discussions are limited to the $M$-band uniform subband coder shown in Fig. 1(a), where the filters are allowed to have infinite order. In Sections II and III, we derive some necessary conditions for optimality in such an orthonormal subband coder. These are called the total-decorrelation condition and the majorization condition. In Section IV, we will show that these two conditions together are, in fact, also sufficient for optimality. In Section V, optimal compaction filters [14]-[18] are first reviewed, and some new viewpoints and properties pointed out. We show in Section VI how the coding gain optimality conditions can be satisfied by designing the analysis

Manuscript received October 2, 1995; revised December 3, 1997. This work was supported in part by the NSF under Grants MIP 92-15785 and MIP 0703755, Tektronix, Inc., and Rockwell International. The associate editor coordinating the review of this paper and approving it for publication was Dr. Truong Q. Nguyen.

The author is with the Department of Electrical Engineering, California Institute of Technology, Pasadena, CA 91125 USA.

Publisher Item Identifier S 1053-587X(98)03915-4.

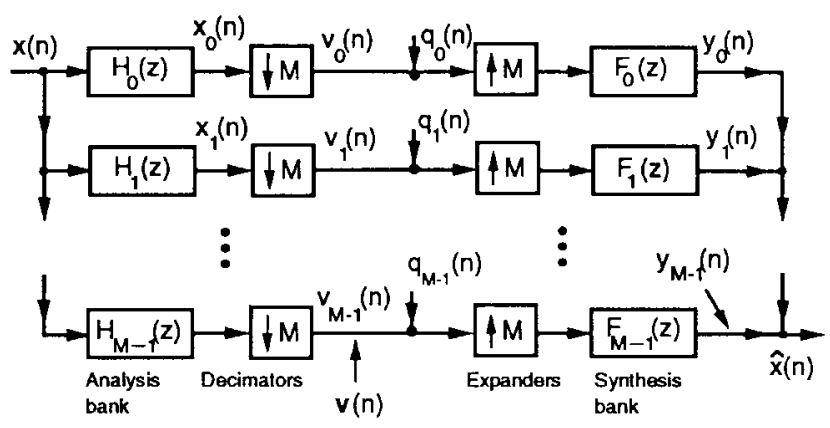

(a)

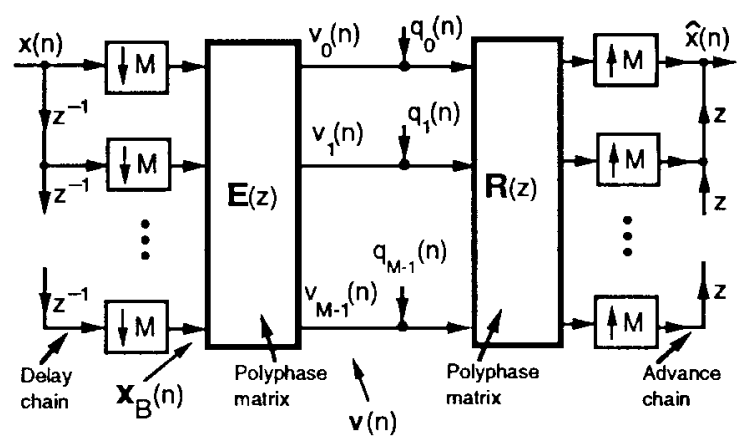

(b)

Fig. 1. (a) Maximally decimated subband coder schematic. (b) Polyphase representation.

filters to be optimum compaction filters for appropriate sets of power spectra derived from the input psd. The optimal filters are ideal and can be identified sequentially one at a time. In Section VII, we study the optimal compaction gain and subband coding gain as functions of $M$ and prove several properties. We show, for example, that these gains are in general not monotone in $M$, unlike the coding gain of the optimal transform coder. In Section VIII, we indicate deeper theoretical differences between various optimal data compression schemes such as the KLT, the optimal subband coder, and predictive coder. The results in these two sections, therefore, complement the parallel results that have been known for transform coders for a long time. The paper concludes by characterizing the class of processes for which orthonormal subband coders are as good as biorthogonal ones (Section IX). Preliminary versions of the results of this paper can be found in two conferences papers [23], [24].

It should be emphasized that the majorization and decorrelation conditions introduced in Sections II and III are necessary and sufficient for optimality only when we do not impose restrictions on the filter orders. Indeed, the optimal filters resulting from the applications of these conditions are of infinite order as seen from the examples of Section VI. In 


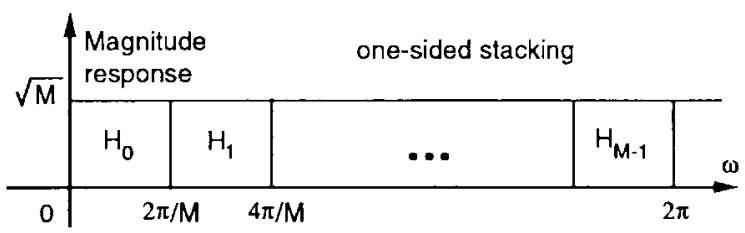

(a)

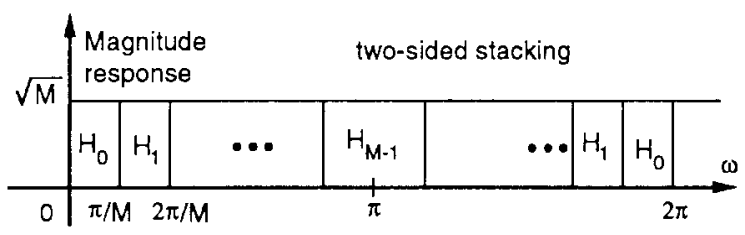

(b)

Fig. 2. Brickwall filter bank with contiguous stacking. (a) One-sided stacking. (b) Two-sided stacking as in the real coefficient case.

general, the optimum solution, e.g., see Fig. 16(i), is different from the contiguous stacking of Fig. 2, which is the traditional subband split. For large number of subbands, the coding gain of the traditional system is not significantly different from the optimal one, but for small $M$, there could be a significant difference. Traditionally, we would approximate the contiguous stacking with finite order, e.g., FIR, filters. One message from the theoretical results of this paper is that if those FIR filters are designed to approximate the optimal stacking instead, then the coding gain will improve. As to how significant this improvement is depends on the specific signal. This is not the topic of discussion here. Perhaps the best way to place the results of this paper in proper context is to regard these as the subband coder version of the optimality results in transform coding theory in the same spirit as Unser's work [15]. While the KLT has long been known to be the optimal transform coder (in the sense of minimum quantization error [1], [2]), the corresponding optimal solution for the uniform subband coder has been explicitly spelt out in this paper. From the results of Section VI, we will see that the optimal filter bank solution is also the principal component filter bank [16], [17] for the given input process. Thus, the principal component idea ties in naturally with the minimization of quantization error due to finite bit rate. In some sense, this should be regarded as a (wonderful) coincidence for uniform filter banks. For nonuniform filter banks, the optimum coding gain problem and the principal component problem do not have identical solutions as shown in [28].

\section{A. Preliminaries}

All results in this paper pertain to the maximally decimated uniform subband coder (identical decimation ratio $M$ in all subbands) shown in Fig. 1(a). The associated polyphase representation [8] is shown in Fig. 1(b). The filter bank is said to be a biorthogonal or perfect reconstruction filter bank if $\mathbf{R}(z) \mathbf{E}(z)=\mathbf{I}$. It is said to be orthonormal or paraunitary if $\mathbf{E}\left(e^{j \omega}\right)$ is unitary for all $\omega$. In the orthonormal case, the perfect reconstruction condition is $F_{i}\left(e^{j \omega}\right)=H_{i}^{*}\left(e^{j \omega}\right)$. In terms of the filters, we can express biorthogonality and orthonormality as [8]

$$
\begin{array}{ll}
\left.H_{k}\left(e^{j \omega}\right) F_{m}\left(e^{j \omega}\right)\right|_{\downarrow M}=\delta(k-m) & \text { (biorthogonality) } \\
\left.H_{k}\left(e^{j \omega}\right) H_{m}^{*}\left(e^{j \omega}\right)\right|_{\downarrow M}=\delta(k-m) & \text { (orthonormality) }
\end{array}
$$

where $\left.F\left(e^{j \omega}\right)\right|_{\downarrow M}$ denotes the Fourier transform of $f(M n)$. The orthonormality condition implies in particular that each filter $H_{i}\left(e^{j \omega}\right)$ satisfies the Nyquist(M) constraint $\left.\left|H_{i}\left(e^{j \omega}\right)\right|^{2}\right|_{\downarrow M}=1$. Traditional contiguous-stackings of brickwall filters (Fig. 2) serve as examples of orthonormal filter banks. In these two examples, each filter is an $\operatorname{aliasfree}(M)$ filter. An aliasfree $(M)$ or antialias $(M)$ filter is defined to be one whose output can be decimated without aliasing, that is, the shifted versions $H_{i}\left[e^{j(\omega-2 \pi k / M)}\right]$ do not overlap for distinct $k$ in $0 \leq k \leq M-1$. Equivalently, we say that the filters have aliasfree $(M)$ supports. Such a support could have multiple number of passbands, e.g., see Fig. 12(b).

1) Statistical Model: The input $x(n)$ is assumed to be zeromean wide sense stationary (WSS) with power spectral density (psd) $S_{x x}\left(e^{j \omega}\right)$. The subband signals $v_{i}(n)$ [see Fig. 1(a)] are therefore (zero-mean and) jointly WSS, and the variances of $x_{i}(n)$ and $v_{i}(n)$ are the same, that is, $\sigma_{x_{i}}^{2}=\sigma_{v_{i}}^{2}$. To derive the coding gain expression, we model the quantizers with additive noise sources $q_{i}(n)$ [Fig. 1(a)]. We assume these noise sources to be jointly WSS with zero mean and variances of the form [3], [8]

$$
\sigma_{q_{i}}^{2}=c 2^{-2 b_{i}} \sigma_{x_{i}}^{2}=c 2^{-2 b_{i}} \sigma_{v_{i}}^{2}
$$

where $b_{i}$ is the number of bits assigned to the $i$ th subband quantizer. The constant $c$ (which depends on the nature of the pdf of the quantizer input [3]) is assumed to be the same for all subbands. The preceding noise model is referred to as the standard noise model in this paper. This model does not require that each $q_{i}(n)$ be white or that any two noise sources be uncorrelated. (For the more general case of biorthogonal filter banks, the white uncorrelated noise model is required in the coding gain derivation [22].)

2) Coding Gain: The quantity $b=\sum_{i=0}^{M-1} b_{i} / M$, which is the average bit rate, is assumed to be fixed. The coding gain of a subband coder is defined by comparing the average mean square value $\mathcal{E}_{S B C}$ of the reconstruction error $x(n)-\hat{x}(n)$ with the m.s. value $\mathcal{E}_{\text {direct }}$ of the direct quantization error (roundoff quantizer) with the same bit rate $b$. Using the standard noise model, an expression for the coding gain $G_{S B C}(M)$ of the orthonormal subband coder of Fig. 1(a) can be derived [8] as

$$
G_{S B C}(M) \triangleq \frac{\mathcal{E}_{\text {direct }}}{\mathcal{E}_{S B C}}=\frac{\sum_{i=0}^{M-1} \sigma_{x_{i}}^{2} / M}{\left(\prod_{i=0}^{M-1} \sigma_{x_{i}}^{2}\right)^{1 / M}}=\frac{\sigma_{x}^{2}}{\left(\prod_{i=0}^{M-1} \sigma_{x_{i}}^{2}\right)^{1 / M}}
$$

This expression assumes optimal bit allocation as described in [3] or [8]. Here, we have used the result $\sum_{i=0}^{M-1} \sigma_{x_{i}}^{2}=M \sigma_{x}^{2}$, which is valid for orthonormal filter banks. The preceding coding gain is the ratio of the arithmetic and geometric means, $A M / G M$ ratio, of the subband variances $\sigma_{x_{i}}^{2}$. For fixed input 


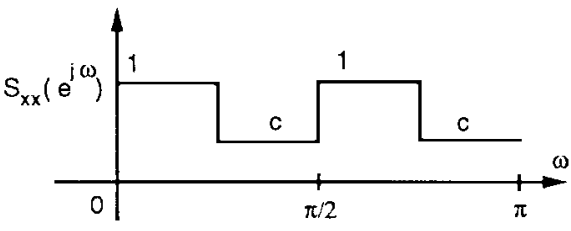

(a)

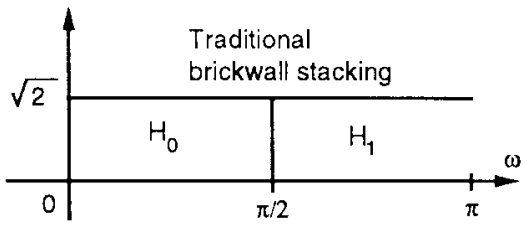

(b)

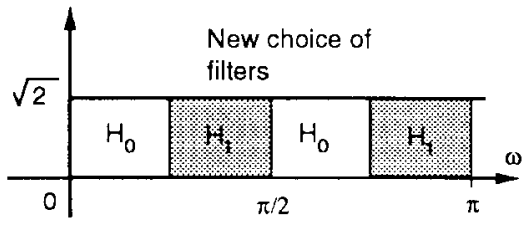

(c)

Fig. 3. Orthonormal filter bank that is better than traditional brickwall stacking. (a) Input psd. (b) Traditional brickwall stacking. (c) New choice of filters.

psd $S_{x x}\left(e^{j \omega}\right)$, the variances $\sigma_{x_{i}}^{2}$ depend only on the analysis filters $H_{i}\left(e^{j \omega}\right)$. We say that the subband coder is optimal [for the fixed $M$ and $S_{x x}\left(e^{j \omega}\right)$ ], if these filters are such that the coding gain is maximized. From (3), we see that coding gain optimization is equivalent to minimizing the product of subband variances.

To demonstrate that traditional contiguous brickwall stacking does not necessarily provide the best coding gain, consider the psd $S_{x x}\left(e^{j \omega}\right)$ shown in Fig. 3(a), with $c>0$. For the traditional two channel analysis filters [Fig. 3(b)], the subband variances are $\sigma_{x_{0}}^{2}=\sigma_{x_{1}}^{2}=(1+c) / 2$, and the coding gain is $G_{S B C}(2)=1$. The second choice of filters shown in Fig. 3(c) has two passbands, but the orthogonality condition [1(b)] still holds. The subband variances are $\sigma_{x_{0}}^{2}=1$ and $\sigma_{x_{1}}^{2}=c$ so that

$$
G_{S B C}(2)=\frac{A M\left\{\sigma_{x_{i}}^{2}\right\}}{G M\left\{\sigma_{x_{i}}^{2}\right\}}=(1+c) / 2 \sqrt{c}>1
$$

For example, if $c=0.01$, we have $G_{S B C}(2)=5.05$, which is a substantial coding gain! Thus, a simple relocation of the passband regions of the analysis filters has improved the coding gain significantly. This new choice is in fact optimal, i.e., it satisfies the sufficient conditions of Section IV.

\section{Total Decorrelation of Subbands}

In orthogonal transform coding theory [where $\mathbf{E}(z)$ in Fig. 1(b) is a constant unitary matrix], it is well known that decorrelation of the decimated subband random variables $v_{i}(n)$ is necessary and sufficient for optimality. That is, the coding gain is maximum if and only if $E\left[v_{i}(n) v_{k}^{*}(n)\right]=0$ for $i \neq k$ and for all $n$. For orthonormal subband coders, a stronger condition is necessary.

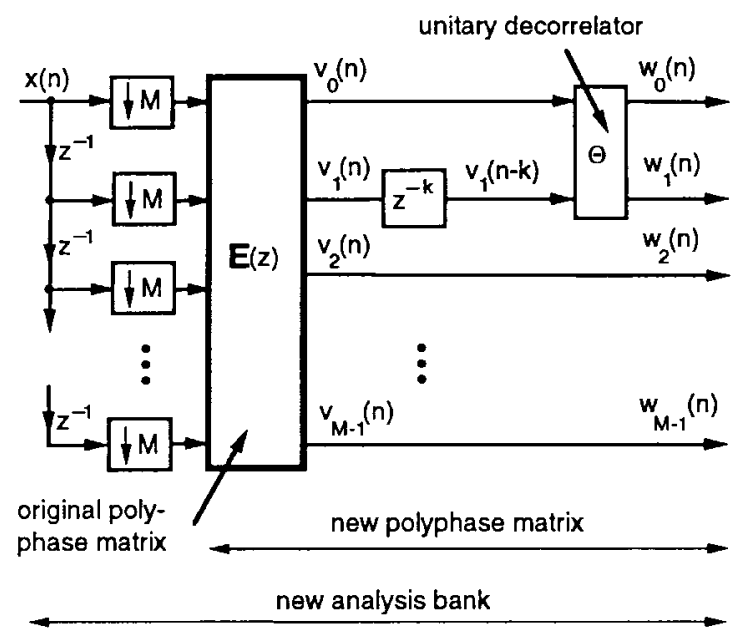

Fig. 4. Increasing the coding gain by exploiting residual correlation.

Theorem 1-Total Decorrelation Is Necessary: For fixed input psd $S_{x x}\left(e^{j \omega}\right)$ and fixed $M$, suppose an orthonormal subband coder is optimal (in the coding gain sense) among the class of all $M$-band orthonormal subband coders. Then, the decimated subband random processes are uncorrelated, that is

$$
E\left[v_{i}(n) v_{k}^{*}(m)\right]=0
$$

for $i \neq k$, and for all $n, m$. This condition will also be referred to as total decorrelation of subbands.

Thus, for optimality, the subband random processes $v_{i}($.$) and v_{k}($.$) must be decorrelated and not just the$ random variables $v_{i}(n)$ and $v_{k}(n)$ for each fixed $n$. Equivalently, the power spectrum matrix of the vector process $\mathbf{v}(n) \triangleq\left[\begin{array}{llll}v_{0}(n) & v_{1}(n) & \cdots & v_{M-1}(n)\end{array}\right]^{T}$ (Fig. 1) must be diagonal. Thus

$$
\mathbf{S}_{\mathrm{vv}}\left(e^{j \omega}\right)=\left[\begin{array}{cccc}
S_{0}\left(e^{j \omega}\right) & 0 & \cdots & 0 \\
0 & S_{1}\left(e^{j \omega}\right) & \cdots & 0 \\
\vdots & \vdots & \ddots & \vdots \\
0 & 0 & \cdots & S_{M-1}\left(e^{j \omega}\right)
\end{array}\right]
$$

where $S_{i}\left(e^{j \omega}\right)$ is the psd of $v_{i}(n)$. Even this stronger condition is not sufficient for optimality. For example, the traditional brickwall subband coder in Fig. 3(b) decorrelates the subband processes trivially, but the coding gain is not optimal.

Proof of Theorem 1: Suppose a pair of decimated subband processes, say $v_{0}($.$) and v_{1}($.$) , are not uncorrelated. Then,$ $E\left[v_{0}(n) v_{1}^{*}(n-k)\right] \neq 0$ for some $k$ (and for all $n$ by the WSS property). We show how to increase the coding gain without violating orthonormality. Suppose we use a delay $z^{-k}$ and a unitary matrix $\Theta$ to transform the pair $v_{0}(n), v_{1}(n-k)$ into an uncorrelated pair $w_{0}(n)$ and $w_{1}(n)$ (Fig. 4). This can be done by choosing $\Theta$ to be the KLT matrix for the vector process $\left[\begin{array}{ll}v_{0}(n) & v_{1}(n-k)\end{array}\right]^{T}$. (A fixed $\Theta$ works for all $n$ by the WSS property.) Thus, we have a modified analysis bank with polyphase matrix

$$
\mathbf{E}_{\text {new }}(z)=\left[\begin{array}{cc}
\boldsymbol{\Theta} & \mathbf{0} \\
\mathbf{0} & \mathbf{I}
\end{array}\right] \boldsymbol{\Lambda}(z) \mathbf{E}(z)
$$

where $\boldsymbol{\Lambda}(z)$ is diagonal, with elements $\left\{1, z^{-k}, 1 \cdots, 1\right\}$ on the diagonal. Since the matrices $\mathbf{E}(z), \boldsymbol{\Lambda}(z)$, and $\boldsymbol{\Theta}$ are 


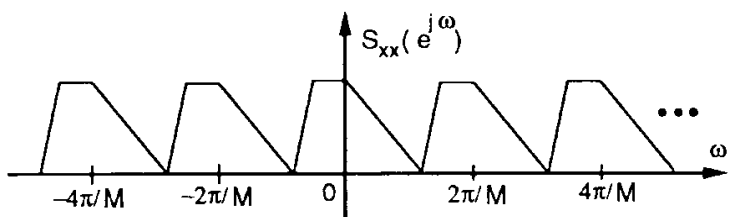

(a)

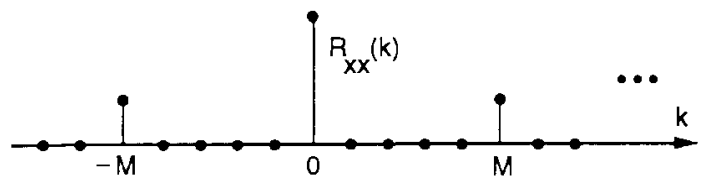

(b)

Fig. 5. (a) Power spectrum for which $M$-band orthonormal subband coders yield no coding gain. (b) Corresponding autocorrelation sequence.

paraunitary, we still have an orthonormal subband coder. We can conclude that the coding gain (3) has been increased if we show that the product of subband variances has been reduced, i.e., $\sigma_{w_{0}}^{2} \sigma_{w_{1}}^{2}<\sigma_{v_{0}}^{2} \sigma_{v_{1}}^{2}$. Let $\mathbf{R}_{w}$ and $\mathbf{R}_{v}$ be the correlation matrices of the vectors $\left[w_{0}(n) \quad w_{1}(n)\right]^{T}$ and $\left[v_{0}(n) \quad v_{1}(n-\right.$ $k)]^{T}$. Then

$$
\mathbf{R}_{w}=\Theta_{\mathbf{R}_{v}} \Theta^{\dagger}
$$

Notice that the diagonal elements of $\mathbf{R}_{v}$ and $\mathbf{R}_{w}$ are the quantities $\sigma_{v_{i}}^{2}$ and $\sigma_{w_{i}}^{2}$. We now have

$$
\sigma_{w_{0}}^{2} \sigma_{w_{1}}^{2}=\operatorname{det} \mathbf{R}_{w}=\operatorname{det} \mathbf{R}_{v}<\sigma_{v_{0}}^{2} \sigma_{v_{1}}^{2} .
$$

The first equality follows because $\mathbf{R}_{w}$ is diagonal [since $w_{0}(n)$ and $w_{1}(n)$ are uncorrelated zero-mean signals]. The second equality follows from unitariness of $\Theta$. The inequality $\operatorname{det} \mathbf{R}_{v}<\sigma_{v_{0}}^{2} \sigma_{v_{1}}^{2}$ follows since det $\mathbf{R}_{v}=\sigma_{v_{0}}^{2} \sigma_{v_{1}}^{2}-|r|^{2}$, where $r$ is the nonzero cross correlation between $v_{0}(n)$ and $v_{1}(n-k)$. Therefore, $\sigma_{w_{0}}^{2} \sigma_{w_{1}}^{2}<\sigma_{v_{0}}^{2} \sigma_{v_{1}}^{2}$ indeed.

$\nabla \nabla \nabla$

Corollary 1-Unity Coding Gain in Orthonormal SBC: The optimal orthonormal subband coder has $G_{S B C}(M)=1$ if and only if the (zero-mean WSS) input process $x(n)$ has power spectral density of the form $S_{x x}\left(e^{j \omega}\right)=S\left(e^{j \omega M}\right)$. When the psd has this form, all the decimated subband signals $v_{i}(n)$ have identical psd $S\left(e^{j \omega}\right)$.

Note: The condition $S_{x x}\left(e^{j \omega}\right)=S\left(e^{j \omega M}\right)$ is equivalent to the condition that the autocorrelation $R_{x x}(k)$ of $x(n)$ is nonzero only when $k$ is a multiple of $M$ (see Fig. 5). Thus, there exist nonwhite inputs for which orthonormal SBC might yield no gain for some fixed $M$. However, unless the input is white, this will not happen for all values of $M$ [because it would mean that $S_{x x}\left(e^{j \omega}\right)$ has a period $2 \pi / M$ for all $\left.M\right]$.

Proof of Corollary 1: If $S_{x x}\left(e^{j \omega}\right)=S\left(e^{j \omega M}\right)$, the psd of the decimated subband signal $v_{i}(n)$ is given by

$$
\begin{aligned}
{\left[l\left(S_{x x}\left(e^{j \omega}\right)\left|H_{i}\left(e^{j \omega}\right)\right|^{2}\right]_{\downarrow M}\right.} & =\left[S\left(e^{j \omega M}\right)\left|H_{i}\left(e^{j \omega}\right)\right|^{2}\right]_{\downarrow M} \\
& =S\left(e^{j \omega}\right)\left[\left|H_{i}\left(e^{j \omega}\right)\right|^{2}\right]_{\downarrow M} \\
& =S\left(e^{j \omega}\right) .
\end{aligned}
$$

The last equality follows from the $\operatorname{Nyquist}(M)$ property of $\left|H_{i}\left(e^{j \omega}\right)\right|^{2}$ that is valid for any $M$-band orthonormal FB (Section I-A). Thus, all the subbands have identical psd, proving $G_{S B C}(M)=1$. Conversely, assume the best possible

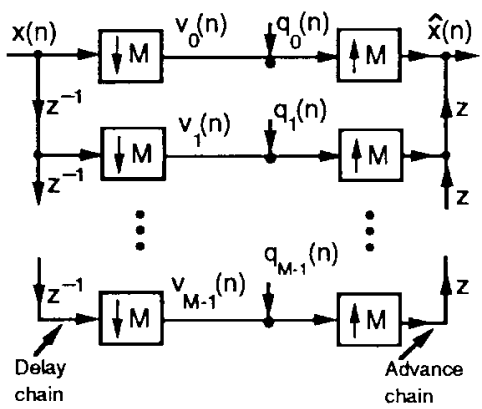

Fig. 6. Delay chain system. Ultra-simple orthonormal filter bank.

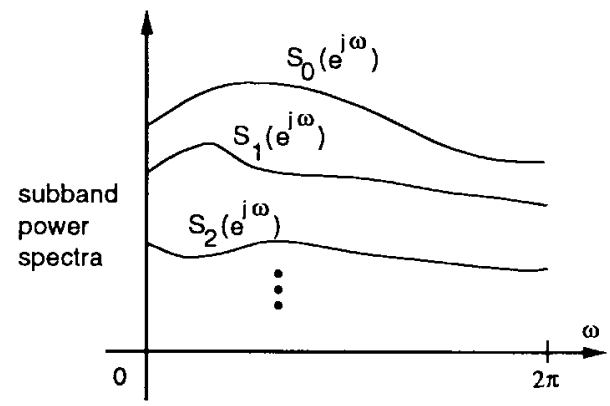

Fig. 7. Demonstration of the majorization property of the decimated subband signals.

coding gain is unity. The delay chain system of Fig. 6, which is a trivial orthonormal filter bank, "achieves" this gain and is therefore optimal. The decimated subband signals here are $v_{i}(n)=x(M n-i), 0 \leq i \leq M-1$. Optimality implies that these random processes are uncorrelated (Theorem 1). In particular $R_{x x}(M n-k)=E\left[x(0) x^{*}(M n-k)\right]=0$ for all $n$ and for $1 \leq k \leq M-1$. Thus, the autocorrelation of $\{x(n)\}$ satisfies $R_{x x}(i)=0$ if $i \neq$ mul. of $M$, and the psd has the form $S_{x x}\left(e^{j \omega}\right)=S\left(e^{j \omega M}\right)$.

$\nabla \nabla \nabla$

\section{THE MAJORIZATION PROPERTY}

Let $S_{k}\left(e^{j \omega}\right)$ denote the power spectrum of the $k$ th decimated subband signal $v_{k}(n)$ in Fig. 1 and $\sigma_{v_{k}}^{2}$ its variance. Assume that the subbands have been numbered such that

$$
\sigma_{v_{0}}^{2} \geq \sigma_{v_{1}}^{2} \geq \cdots \geq \sigma_{v_{M-1}}^{2}
$$

[Note that $\sigma_{v_{k}}^{2}=\sigma_{x_{k}}^{2}$ in Fig. 1(a).] We say that the set of subband signals, or the set of subband power spectra $\left\{S_{k}\left(e^{j \omega}\right)\right\}$, has the majorization property if

$$
S_{0}\left(e^{j \omega}\right) \geq S_{1}\left(e^{j \omega}\right) \geq \cdots \geq S_{M-1}\left(e^{j \omega}\right) \quad \text { for all } \omega .
$$

That is, the $k$ th subband psd dominates the $(k+1)$ th psd for all $\omega$. This is demonstrated in Fig. 7. We sometimes say that the set of decimated random processes $\left\{v_{k}(n)\right\}$ is majorized.

Theorem 2-Majorization Is Necessary: For fixed input psd $S_{x x}\left(e^{j \omega}\right)$ and fixed $M$, suppose an orthonormal subband coder is optimal (in the coding gain sense) among the class of all $M$-band orthonormal subband coders. Then, the decimated subband signals $v_{k}(n)$ have the majorization property. 


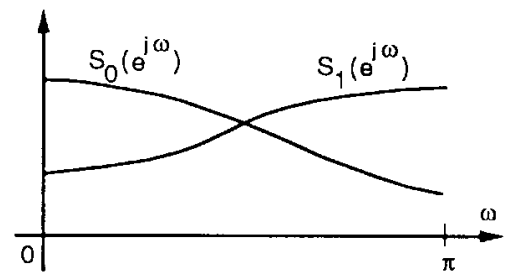

Fig. 8. Example of the power spectra of two decimated subbands. One psd dominates in some places, and the other in some places, showing that the subbands are not majorized.

Proof: Assuming that the majorization property is not satisfied, we will show how the coding gain can be increased without violating orthonormality. For example, assume $S_{0}\left(e^{j \omega}\right) \geq S_{1}\left(e^{j \omega}\right)$ is not valid for all $\omega$, even though $\sigma_{v_{0}}^{2} \geq \sigma_{v_{1}}^{2}$. That is, for some values of $\omega, S_{0}\left(e^{j \omega}\right)$ is larger, and for some other values of $\omega, S_{1}\left(e^{j \omega}\right)$ is larger (Fig. 8). Let $\mathbf{E}\left(e^{j \omega}\right)$ denote the polyphase matrix of the orthonormal filter bank. Let us cascade a matrix $\mathbf{T}\left(e^{j \omega}\right)$ as shown in Fig. 9. We will choose $\mathbf{T}\left(e^{j \omega}\right)$ as

$$
\mathbf{T}\left(e^{j \omega}\right)=\left\{\begin{array}{cl}
\mathbf{I}_{M}, & \\
{\left[\begin{array}{ccc}
0 & 1 & \mathbf{0} \\
1 & 0 & \mathbf{0} \\
\mathbf{0} & \mathbf{0} & \mathbf{I}_{M-2}
\end{array}\right],} & \text { if } S_{0}\left(e^{j \omega}\right) \geq S_{1}\left(e^{j \omega}\right) \\
& \text { if } S_{0}\left(e^{j \omega}\right)<S_{1}\left(e^{j \omega}\right) .
\end{array}\right.
$$

The new pair of power spectra $S_{0}^{\prime}\left(e^{j \omega}\right), S_{1}^{\prime}\left(e^{j \omega}\right)$ will then satisfy the property $S_{0}^{\prime}\left(e^{j \omega}\right) \geq S_{1}^{\prime}\left(e^{j \omega}\right)$ for all $\omega$. Moreover, for each $\omega, S_{0}^{\prime}\left(e^{j \omega}\right) \geq S_{0}\left(e^{j \omega}\right)$, whereas $S_{1}^{\prime}\left(e^{j \omega}\right) \leq S_{1}\left(e^{j \omega}\right)$. Thus, the variances of the new signals $w_{0}(n)$ and $w_{1}(n)$ are such that $\sigma_{w_{0}}^{2} \geq \sigma_{v_{0}}^{2}$ and $\sigma_{w_{1}}^{2} \leq \sigma_{v_{1}}^{2}$. Since $\mathbf{T}\left(e^{j \omega}\right)$ is paraunitary by construction, the filter bank remains orthonormal, and the sum of variances is preserved, that is, $\sigma_{w_{0}}^{2}+\sigma_{w_{1}}^{2}=\sigma_{v_{0}}^{2}+\sigma_{v_{1}}^{2}$. Thus, we can write

$$
\sigma_{w_{0}}^{2}=\sigma_{w_{0}}^{2}+\delta, \quad \sigma_{w_{1}}^{2}=\sigma_{v_{1}}^{2}-\delta, \quad \delta>0
$$

so that $\sigma_{w_{0}}^{2} \sigma_{w_{1}}^{2}=\sigma_{v_{0}}^{2} \sigma_{v_{1}}^{2}-\delta^{2}-\delta\left(\sigma_{v_{0}}^{2}-\sigma_{v_{1}}^{2}\right)$. Since $\delta>0$ and the convention $\sigma_{v_{0}}^{2} \geq \sigma_{v_{1}}^{2}$ is in effect, the preceding equation implies $\sigma_{w_{0}}^{2} \sigma_{w_{1}}^{2}<\sigma_{v_{0}}^{2} \sigma_{v_{1}}^{2}$; therefore, the coding gain is increased.

$\nabla \nabla \nabla$

\section{A Set of NeCESSARY AND SufFicient CONDITIONS}

Notice first that the simple delay chain filter bank (Fig. 6), which yields no coding gain for any input psd, satisfies the majorization property (since all subbands have identical psd). On the other hand, the brickwall subband coder with contiguous stacking, e.g., Fig. 3(b), satisfies the total decorrelation property for any input psd, showing that it is not sufficient for optimality. Thus, although majorization and decorrelation are necessary for the optimality of an orthonormal filter bank, neither of them is individually sufficient. However, if we put them together, that turns out to be sufficient!

Theorem 3-A Necessary and Sufficient Condition for Optimality: The coding gain of an $M$-band orthonormal filter bank is maximum (among all $M$ band orthonormal subband coders) for a given input psd $S_{x x}\left(e^{j \omega}\right)$ if and only if the decimated subband signals $v_{k}(n)$ satisfy the following two properties:

1) They are totally uncorrelated, that is, $E\left[v_{i}(n) v_{k}^{*}(m)\right]=$ $0, i \neq k$ for all $m, n$.

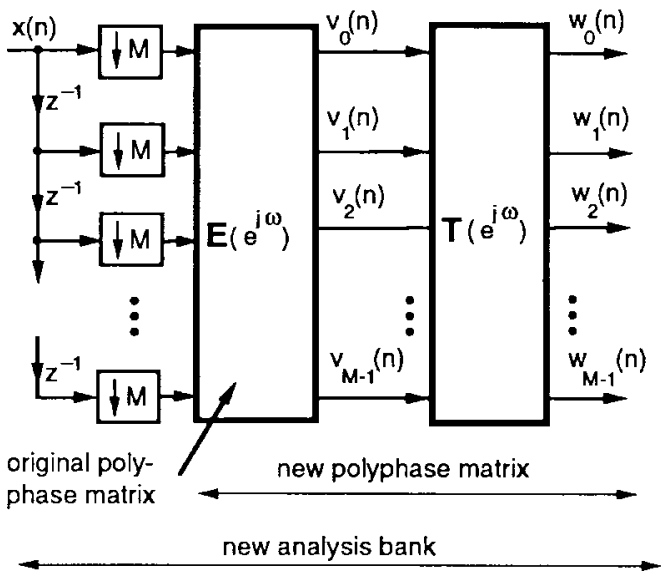

Fig. 9. Increasing the coding gain by exploiting unmajorized subband psd.

2) They are majorized, that is, the power spectra satisfy $S_{0}\left(e^{j \omega}\right) \geq S_{1}\left(e^{j \omega}\right) \geq \cdots \geq S_{M-1}\left(e^{j \omega}\right)$ (assuming the subbands are numbered appropriately).

Furthermore, when these conditions are satisfied, the set of power spectra $\left\{S_{k}\left(e^{j \omega}\right)\right\}$ of the decimated subband signals is unique, although the analysis filters that yield these may not be unique.

Proof: In view of earlier theorems, it only remains to prove that total decorrelation and majorization together imply optimality. From Fig. 1(b), we have $\mathbf{S}_{\mathbf{v v}}\left(e^{j \omega}\right)=$ $\mathbf{E}\left(e^{j \omega}\right) \mathbf{S}_{B}\left(e^{j \omega}\right) \mathbf{E}^{\dagger}\left(e^{j \omega}\right)$, where $\mathbf{S}_{B}\left(e^{j \omega}\right)$ and $\mathbf{S}_{\mathbf{v v}}\left(e^{j \omega}\right)$ are power spectra of the vectors $\mathrm{x}_{B}(n)$ and $\mathbf{v}(n)$ indicated in the figure. Note that $\mathbf{x}_{B}(n)$ is the $M$-fold blocked version [8] of the input $x(n)$. If $\mathbf{E}\left(e^{j \omega}\right)$ performs total decorrelation, $\mathbf{S}_{\mathbf{v v}}\left(e^{j \omega}\right)$ is diagonal.

$$
\begin{aligned}
\mathbf{S}_{\mathbf{v v}}\left(e^{j \omega}\right) & =\mathbf{E}\left(e^{j \omega}\right) \mathbf{S}_{B}\left(e^{j \omega}\right) \mathbf{E}^{\dagger}\left(e^{j \omega}\right) \\
& =\operatorname{diag}\left\{S_{0}\left(e^{j \omega}\right) S_{1}\left(e^{j \omega}\right) \cdots S_{M-1}\left(e^{j \omega}\right)\right\} .
\end{aligned}
$$

Since $\mathbf{E}\left(e^{j \omega}\right)$ is unitary, this implies that for each fixed $\omega$, the subband power spectra $S_{k}\left(e^{j \omega}\right)$ are eigenvalues of $\mathbf{S}_{B}\left(e^{j \omega}\right)$. Suppose the majorization property also holds. This means that for each $\omega$, these eigenvalues are ordered in a decreasing fashion. Since the set of eigenvalues is unique, each diagonal element in (15) is uniquely determined for each $\omega$. Thus, the set of power spectra $\left\{S_{k}\left(e^{j \omega}\right)\right\}$, which has the majorization property, is unique as claimed in the theorem. [However, since the eigenvector matrix $\mathbf{E}^{\dagger}\left(e^{j \omega}\right)$ may not be unique, the analysis bank may not be unique for a given input psd.] Since majorization and total decorrelation are necessary for optimality and since there is only one set of majorized decorrelated subband power spectra, it follows that majorization together with decorrelation leads to optimality.

$\nabla \nabla \nabla$

\section{COMPACTION FILTERS}

The role of the energy compaction concept in subband coding theory has been observed by a number of authors [14]-[21]. Fig. 10 shows a filter $H\left(e^{j \omega}\right)$ with a zero-mean WSS input $x(n)$ having psd $S_{x x}\left(e^{j \omega}\right)$. This can be regarded as an $M$-fold decimation filter, that is, one branch of an 


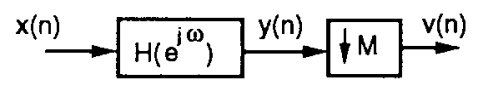

Fig. 10. Pertaining to the optimal energy compaction problem.

$M$-channel analysis bank. Notice that $\sigma_{y}^{2}=\sigma_{v}^{2}$ in Fig. 10 . Consider the problem of designing $H\left(e^{j \omega}\right)$ such that the output variance $\sigma_{y}^{2}$ is maximized subject to the constraint that $\left|H\left(e^{j \omega}\right)\right|^{2}$ be Nyquist $(M)$, that is

$$
\begin{array}{r}
\left.\left|H\left(e^{j \omega}\right)\right|^{2}\right|_{\downarrow M}=1, \quad \text { i.e., } \quad \sum_{k=0}^{M-1}\left|H\left[e^{j(\omega-2 \pi k / M)}\right]\right|^{2}=M \\
\text { for all } \omega \text { (Nyquist constraint). }
\end{array}
$$

The solution $H\left(e^{j \omega}\right)$ will be called an optimum compaction filter. The Nyquist constraint is imposed because $\left|H_{k}\left(e^{j \omega}\right)\right|^{2}$ is $\operatorname{Nyquist}(M)$ for every analysis filter $H_{k}\left(e^{j \omega}\right)$ in any orthonormal filter bank. Notice that the Nyquist constraint implies the unit-energy property $\int_{0}^{2 \pi}\left|H\left(e^{j \omega}\right)\right|^{2} d \omega / 2 \pi=1$ as well as the boundedness property $\left|H\left(e^{j \omega}\right)\right|^{2} \leq M$. The following result is a refined version for arbitrary $M$ of Unser's construction of compaction filters [14].

Theorem 4-Optimum Compaction Filters: Consider the following construction of $H\left(e^{j \omega}\right)$.

Step 1) For each frequency $\omega_{0}$ in $0 \leq \omega<2 \pi / M$, define the $M$ alias frequencies $\omega_{k}=\omega_{0}+2 \pi k / M$, where $0 \leq k \leq M-1$.

Step 2) Compare the values of $S_{x x}\left(e^{j \omega}\right)$ at these $M$ alias frequencies $\left\{\omega_{k}\right\}$. Let $L$ be the smallest integer such that $S_{x x}\left(e^{j \omega_{L}}\right)$ is a maximum in this set. Then, assign

$H\left\{e^{j\left[\omega_{0}+(2 k \pi / M)\right]}\right\}= \begin{cases}\sqrt{M}, & \text { when } k=L \\ 0, & \text { otherwise. }\end{cases}$

Repeating this for each $\omega_{0}$ in the region $0 \leq \omega<$ $2 \pi / M$, the filter $H\left(e^{j \omega}\right)$ is completely defined for all $\omega$ in $0 \leq \omega<2 \pi$. This filter satisfies the Nyquist $(M)$ constraint (16) and, moreover, maximizes the output variance $\sigma_{v}^{2}$ under this constraint.

While the optimal compaction filter is not unique (see below), the above construction has the following special properties.

a) $H\left(e^{j \omega}\right)$ is an ideal two-level filter with passband response $=\sqrt{M}$ and stopband response $=0$.

b) $H\left(e^{j \omega}\right)$ is an $\operatorname{antialias}(M)$ filter (Section I-A). This follows because if $H\left(e^{j \omega}\right) \neq 0$ for some $\omega$, then $H\left[e^{j(\omega-2 \pi k / M)}\right]=0$ for $1 \leq k \leq M-1$.

c) The total width of all passbands is $2 \pi / M$.

Examples: Let $M=3$ and the input psd $S_{x x}\left(e^{j \omega}\right)$ be as in Fig. 11(a). First, divide the frequency axis of the psd into three equal regions, numbered as 0,1 , and 2 . If $0 \leq \omega_{0} \leq \alpha$, then $S_{x x}\left(e^{j \omega_{0}}\right)$ dominates the psd at the alias frequencies $\omega_{0}+2 \pi / 3$ and $\omega_{0}+4 \pi / 3$ (which are in regions 1 and 2 , respectively). Therefore, region 0 dominates, but for $\alpha<\omega_{0} \leq 2 \pi / 3$, the value of $S_{x x}\left(e^{j \omega}\right)$ at $\omega=\omega_{0}+4 \pi / 3$ (region 2) dominates the values at the alias frequencies $\omega_{0}$ and $\omega_{0}+2 \pi / 3$ (which are in regions 0 and 1). The optimal compaction filter given by the

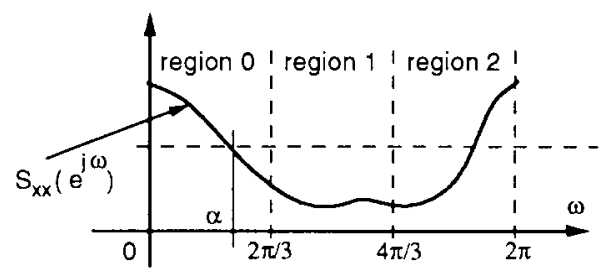

(a)

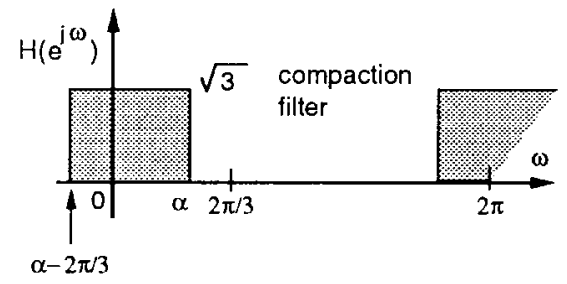

(b)

Fig. 11. (a) Input psd. (b) Optimal compaction filter for this input for $M=3$.

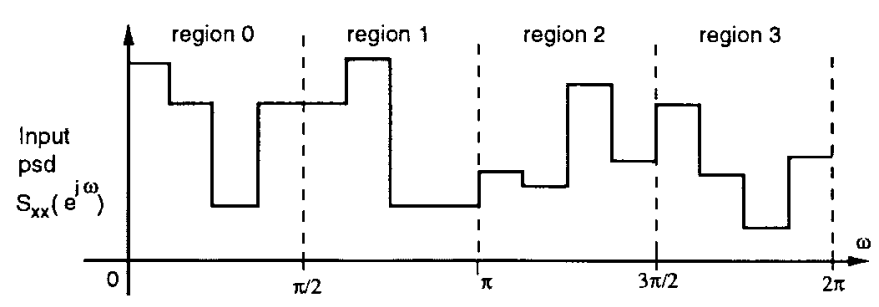

(a)

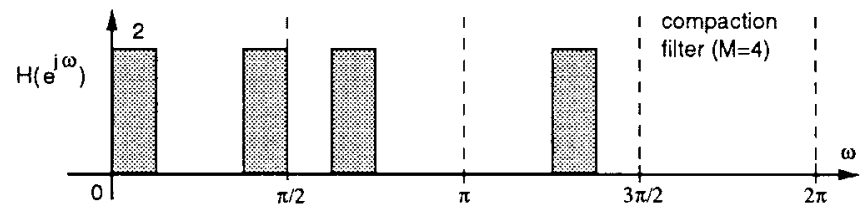

(b)

Fig. 12. (a) Input psd. (b) Corresponding optimal energy compaction filter for $M=4$.

above theorem is as in Fig. 11(b). The Nyquist(3) property of $\left|H\left(e^{j \omega}\right)\right|^{2}$ is easy to verify. Fig. 12 shows another example for $M=4$. The optimum filter here is multiband (rather than lowpass). The Nyquist(4) property of $\left|H\left(e^{j \omega}\right)\right|^{2}$ can be verified with some effort.

Proof of Theorem 4: The psd of $v(n)$ is given by

$$
\begin{aligned}
S_{v v}\left(e^{j \omega}\right) & =\left[S_{x x}\left(e^{j \omega}\right)\left|H\left(e^{j \omega}\right)\right|^{2}\right]_{\downarrow M} \\
& =\frac{1}{M} \sum_{k=0}^{M-1} S_{x x}\left[e^{j(\omega+2 \pi k) / M}\right]\left|H\left[e^{j(\omega+2 \pi k) / M}\right]\right|^{2} .
\end{aligned}
$$

Therefore

$$
\begin{aligned}
\sigma_{v}^{2}= & \int_{0}^{2 \pi} S_{v v}\left(e^{j \omega}\right) d \omega / 2 \pi \\
= & \frac{1}{M} \int_{0}^{2 \pi} \sum_{k=0}^{M-1} S_{x x}\left\{e^{j[\omega+(2 \pi k / M)]}\right\} \\
& \cdot\left|H\left\{e^{j[\omega+(2 \pi k / M)]}\right\}\right|^{2} d \omega / 2 \pi
\end{aligned}
$$


after a minor change of variables. Since the integrand is nonnegative, we only have to maximize

$$
\sum_{k=0}^{M-1} S_{x x}\left\{e^{j[\omega+(2 \pi k / M)]}\right\}\left|H\left\{e^{j[\omega+(2 \pi k / M)]}\right\}\right|^{2}
$$

for each $\omega$ in $0 \leq \omega<2 \pi / M$ by choice of $\left|H\left(e^{j \omega}\right)\right|^{2}$ [the psd $S_{x x}\left(e^{j \omega}\right)$ is fixed]. For this, consider a sum of the form $\boldsymbol{\Psi}=\sum_{k=0}^{M-1} c_{k} \phi_{k}$, where $c_{k}$ are fixed nonnegative numbers such that $c_{0} \geq c_{1} \geq \cdots \geq c_{M-1} \geq 0$, and $\phi_{k} \geq 0$ are to be chosen such that the sum $\boldsymbol{\Psi}$ is maximized under the constraint $\sum_{k=0}^{M-1} \phi_{k}=\phi>0$. Then, the best choice of the constants $\phi_{k}$ is given by $\phi_{0}=\phi$ and $\phi_{k}=0, k>0$. To verify this, write

$$
\begin{aligned}
\Psi= & c_{0} \phi_{0}+\sum_{k=1}^{M-1} c_{k} \phi_{k}=c_{0} \phi-c_{0} \sum_{k=1}^{M-1} \phi_{k} \\
& +\sum_{k=1}^{M-1} c_{k} \phi_{k}=c_{0} \phi+\sum_{k=1}^{M-1}\left(c_{k}-c_{0}\right) \phi_{k} .
\end{aligned}
$$

Since $c_{0} \geq c_{k}$ and $\phi_{k} \geq 0$ for all $k$, the second term is $\leq 0$. The sum $\boldsymbol{\Psi}$ is therefore maximized by the claimed choice. Thus, the quantity (20) is maximized for a fixed $\omega$, say, $\omega=\omega_{0}$, under the $\operatorname{Nyquist}(M)$ constraint if $\left|H\left\{e^{j\left[\omega_{0}+(2 \pi k / M)\right]}\right\}\right|^{2}=M$ for one value of $k$ that yields maximum $S_{x x}\left\{e^{j\left[\omega_{0}+(2 \pi k / M)\right]}\right\}$, and $H\left\{e^{j\left[\omega_{0}+(2 \pi k / M)\right]}\right\}=0$ for all other $k$. This choice evidently satisfies the $\operatorname{Nyquist}(M)$ constraint.

$\nabla \nabla \nabla$

\section{A. Remarks on the Compaction Filter}

We summarize for convenience a number of properties of optimal compaction filters, some of which were also observed by Unser [14]:

1) Nonuniqueness: The magnitude $\left|H\left(e^{j \omega}\right)\right|$ is not unique because of possible ties in the comparison step of Theorem 4. Thus, the solution is not unique. For example, if $x(n)$ is white, any filter satisfying the Nyquist $(M)$ condition is optimal.

2) Invariance to Monotone Transformations: If $H\left(e^{j \omega}\right)$ is an optimal compaction filter for an input psd $S_{x x}\left(e^{j \omega}\right)$, then it will be a valid optimal solution for the modified psd $\sqrt{S_{x x}\left(e^{j \omega}\right)}$ because the results based on the comparisons described in Theorem 4 are not affected. In fact, the compaction filter will remain optimal for any transformed psd $f\left[S_{x x}\left(e^{j \omega}\right)\right]$ as long as $f[] \geq$.0 is a nondecreasing function.

3) Case of Real Inputs: In general, the compaction filter has no symmetry with respect to zero frequency. For a real input $x(n)$, the psd $S_{x x}\left(e^{j \omega}\right)$ is symmetric, and we can modify the construction of Theorem 4 to obtain a symmetric solution $H\left(e^{j \omega}\right)$. This is demonstrated in Fig. 13 for $M=3$.

4) Case of Monotone psd: If a psd is nonincreasing in $[0,2 \pi)$, then the complex lowpass filter with passband $[0,2 \pi / M)$ works as an optimum compaction filter [Fig. 14(a)]. For real random processes with nonincreasing psd in $[0, \pi]$, the real lowpass filter with passband $[-\pi / M, \pi / M)$ is optimum [see Fig. 14(b)].

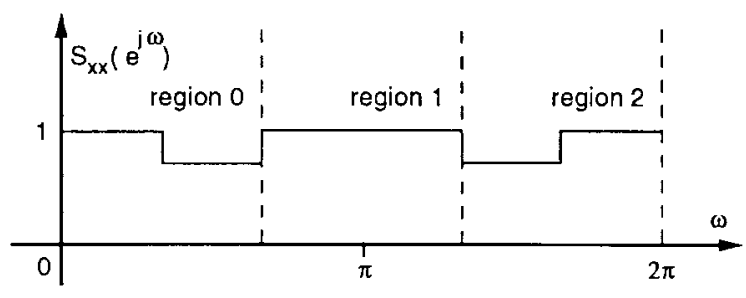

(a)

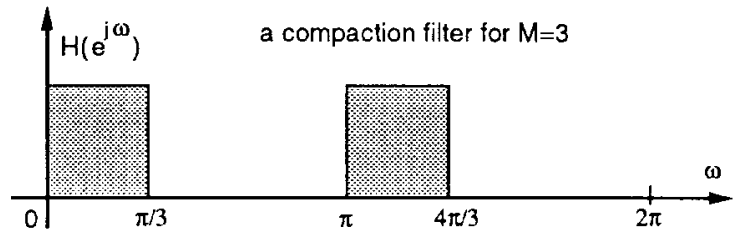

(b)

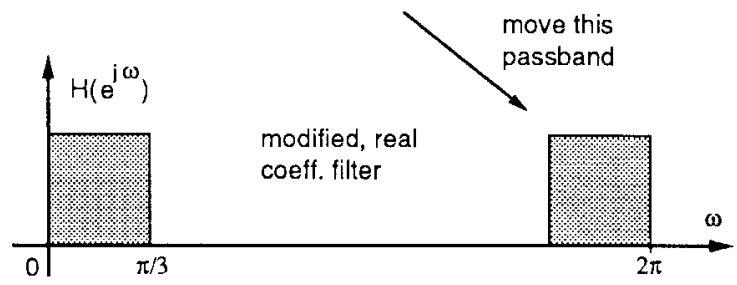

(c)

Fig. 13. (a) Input psd with symmetry. (b) Compaction filter for $M=3$ designed using the Theorem. (c) Real coefficient filter with the same compaction performance.

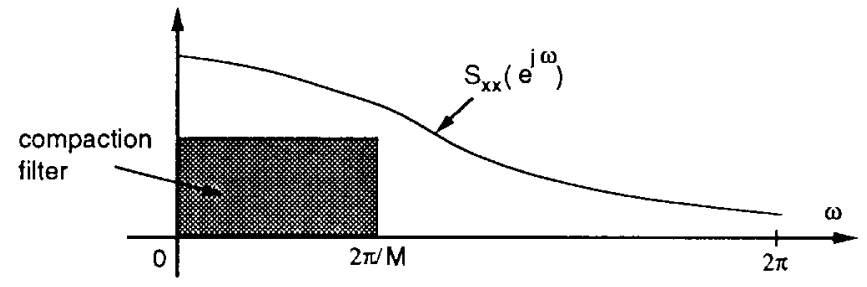

(a)

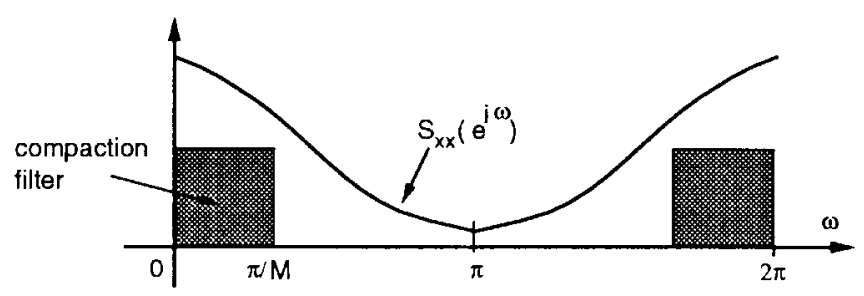

(b)

Fig. 14. (a) Monotone input psd and the optimal compaction filter. (b) Monotone symmetric psd (as for a real process) and the corresponding real coefficient optimal compaction filter.

\section{B. The Energy Compaction Problem}

\section{Formulated as an Eigenproblem}

As shown first by Tsatsanis and Giannakis [17], the compaction problem can be elegantly written as an eigenproblem. For this, we represent $H\left(e^{j \omega}\right)$ in the polyphase form [8] $H(z)=\sum_{k=0}^{M-1} z^{-k} E_{k}\left(z^{M}\right)$ and redraw the compaction filter as in Fig. 15. The vector signal indicated as $\mathbf{x}_{B}(n)$ is the blocked version of $x(n)$. With $\mathbf{S}_{B}\left(e^{j \omega}\right)$ denoting the psd 


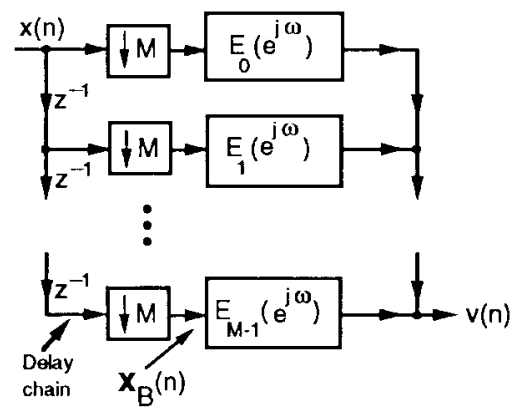

Fig. 15. Compaction filter redrawn in polyphase form.

matrix of this blocked process, the psd of the output $v(n)$ is

$$
\begin{aligned}
S_{v v}\left(e^{j \omega}\right) & =\mathbf{e}^{\dagger}\left(e^{j \omega}\right) \mathbf{S}_{B}\left(e^{j \omega}\right) \mathbf{e}\left(e^{j \omega}\right), \\
\mathbf{e}^{\dagger}\left(e^{j \omega}\right) & \triangleq\left[\begin{array}{lll}
E_{0}\left(e^{j \omega}\right) & E_{1}\left(e^{j \omega}\right) \cdots & E_{M-1}\left(e^{j \omega}\right)
\end{array}\right] .
\end{aligned}
$$

Now, the Nyquist $(M)$ constraint on $\left|H\left(e^{j \omega}\right)\right|^{2}$ is equivalent (see [8, p. 159]) to the power complementary property of the polyphase components, that is, $\sum_{k=0}^{M-1}\left|E_{k}\left(e^{j \omega}\right)\right|^{2}=1$ for all $\omega$. In vector notation

$$
\mathbf{e}^{\dagger}\left(e^{j \omega}\right) \mathbf{e}\left(e^{j \omega}\right)=1 \quad \text { for all } \omega
$$

The variance $\sigma_{v}^{2}$ of the output $v(n)$ is the integral

$$
\begin{aligned}
\sigma_{v}^{2} & =\int_{0}^{2 \pi} S_{v v}\left(e^{j \omega}\right) d \omega / 2 \pi \\
& =\int_{0}^{2 \pi} \mathbf{e}^{\dagger}\left(e^{j \omega}\right) \mathbf{S}_{B}\left(e^{j \omega}\right) \mathbf{e}\left(e^{j \omega}\right) d \omega / 2 \pi .
\end{aligned}
$$

Since the integrand is nonnegative, the optimal compaction problem is equivalent to maximizing the quadratic $\mathbf{e}^{\dagger}\left(e^{j \omega}\right) \mathbf{S}_{B}\left(e^{j \omega}\right) \mathbf{e}\left(e^{j \omega}\right)$ pointwise for each frequency $\omega$, subject to $\mathbf{e}^{\dagger}\left(e^{j \omega}\right) \mathbf{e}\left(e^{j \omega}\right)=1$. However, $\mathbf{S}_{B}\left(e^{j \omega}\right)$ is Hermitian (and positive semidefinite); therefore, the solution $\mathbf{e}\left(e^{j \omega}\right)$ for each $\omega$ is an eigenvector corresponding to the maximum eigenvalue of $\mathbf{S}_{B}\left(e^{j \omega}\right)$. Then, $S_{v v}\left(e^{j \omega}\right)$ is this maximum eigenvalue at the frequency $\omega$, and the maximized variance $\sigma_{v}^{2}$ is the integral of this eigenvalue. Summarizing, we have proved Theorem 5 .

Theorem 5-Compaction Filters from Eigenvectors: Let $\mathbf{S}_{B}\left(e^{j \omega}\right)$ be the $M \times M$ psd matrix of the $M$-fold blocked version $\mathrm{x}_{B}(n)$ of the input $x(n)$ (see Fig. 15). Define the column vector $\mathbf{e}\left(e^{j \omega}\right)$ from the polyphase components of $H\left(e^{j \omega}\right)$ as in (22). Then, the filter $H\left(e^{j \omega}\right)$ is an optimum compaction filter for the input psd $S_{x x}\left(e^{j \omega}\right)$ if and only if, for each $\omega$, the following are true.

1) $\mathbf{e}^{\dagger}\left(e^{j \omega}\right) \mathbf{e}\left(e^{j \omega}\right)=1$ [equivalent to $\operatorname{Nyquist}(M)$ constraint on $\left.\left|H\left(e^{j \omega}\right)\right|^{2}\right]$.

2) $\mathbf{e}\left(e^{j \omega}\right)$ is an eigenvector of $\mathbf{S}_{B}\left(e^{j \omega}\right)$ corresponding to the maximum eigenvalue.

This maximum eigenvalue is the psd $S_{v v}\left(e^{j \omega}\right)$ of the decimated output process $v(n)$. Even though the optimized compaction filter is not unique (because eigenvectors may not be unique), the corresponding output psd $S_{v v}\left(e^{j \omega}\right)$ is unique. $\diamond$

\section{Optimal ORTHONORMAL SUBBAND CODERS}

In this section we will show that the optimal $M$-band orthonormal filter bank for a given input psd can be found by successively solving a set of $M$ optimal energy compaction problems one at a time.

\section{A. Procedure to Design Optimal Orthonormal Filter Banks}

We first describe the procedure with the help of an example and then validate it by supplying the underlying theory. Suppose the given input psd is as in Fig. 16(a). The first step is to choose one filter $H_{0}\left(e^{j \omega}\right)$ to be an optimal energy compaction filter for the input psd $S_{x x}\left(e^{j \omega}\right)$. This filter, which has been designed using Theorem 4, is shown in Fig. 16(b). Let the passband support of $H_{0}\left(e^{j \omega}\right)$ be denoted $\mathcal{S}_{0}$. Suppose we define a new psd

$$
S_{x x}^{(1)}\left(e^{j \omega}\right)= \begin{cases}0 & \text { in } \mathcal{S}_{0} \\ S_{x x}\left(e^{j \omega}\right) & \text { otherwise }\end{cases}
$$

as shown in Fig. 16(c). Thus, $S_{x x}^{(1)}\left(e^{j \omega}\right)$ is obtained by peeling off the portion of $S_{x x}\left(e^{j \omega}\right)$ falling in the passband of $H_{0}\left(e^{j \omega}\right)$. We design the next analysis filter $H_{1}\left(e^{j \omega}\right)$ to be the optimal compaction filter for this partial psd $S_{x x}^{(1)}\left(e^{j \omega}\right)$. This is shown in Fig. 16(d). Define the next partial psd $S_{x x}^{(2)}\left(e^{j \omega}\right)$ by peeling off the portions of $S_{x x}\left(e^{j \omega}\right)$ in the passbands of $H_{0}\left(e^{j \omega}\right)$ and $H_{1}\left(e^{j \omega}\right)$, as demonstrated in Fig. 16(e). We then design $H_{2}\left(e^{j \omega}\right)$ to be the optimal compaction filter for this new partial psd $S_{x x}^{(2)}\left(e^{j \omega}\right)$. Finally, by peeling off the portions of $S_{x x}\left(e^{j \omega}\right)$ that fall under the passbands of $H_{0}\left(e^{j \omega}\right), H_{1}\left(e^{j \omega}\right)$ and $H_{2}\left(e^{j \omega}\right)$, we obtain the partial psd $S_{x x}^{(3)}\left(e^{j \omega}\right)$ and the corresponding compaction filter $H_{3}\left(e^{j \omega}\right)$. In fact, the passband of $H_{3}\left(e^{j \omega}\right)$ is simply the portion of $[0,2 \pi)$ obtained by peeling off the passbands of $H_{0}\left(e^{j \omega}\right), H_{1}\left(e^{j \omega}\right)$ and $H_{2}\left(e^{j \omega}\right)$. Thus, all the analysis filters have been identified [see Fig. 16(i)].

Since all the filters are designed using Theorem 4, they are ideal aliasfree $(M)$ filters. By construction, no two filters overlap, and together, they tile the region $[0,2 \pi)$. Finally, since $H_{k}\left(e^{j \omega}\right) H_{m}\left(e^{j \omega}\right)=0$ for $k \neq m$ and $\left|H_{k}\left(e^{j \omega}\right)\right|^{2}$ is Nyquist $(M)$, the resulting filter bank is orthonormal [i.e., (1b) holds]. Readers familiar with the important concept of principal component filter banks advanced in [16] and [17] will recognize that the preceding construction yields such a filter bank. This was also observed in [21].

Proof of Optimality: The nonoverlapping property of the filters implies total decorrelation of subbands. In view of Theorem 3, it only remains to verify the majorization property. Let $\omega_{0}$ be any frequency in $0 \leq \omega_{0}<2 \pi / M$, and define

$$
\omega_{i}=\omega_{0}+\frac{2 \pi i}{M}, \quad i=0,1, \cdots, M-1 .
$$

These are the $M$ alias-frequencies of $\omega_{0}$. By construction, each filter $H_{k}\left(e^{j \omega}\right)$ has exactly one of these frequencies - call it $\omega_{n_{k}}$-falling in its passband. Since the compaction filters are designed in the sequential order, the condition $H_{0}\left(e^{j \omega}\right), H_{1}\left(e^{j \omega}\right), \cdots, H_{M-1}\left(e^{j \omega}\right)$

$$
S_{x x}\left(e^{j \omega_{n_{0}}}\right) \geq S_{x x}\left(e^{j \omega_{n_{1}}}\right) \geq \cdots \geq S_{x x}\left(e^{j \omega_{n_{M}-1}}\right) .
$$




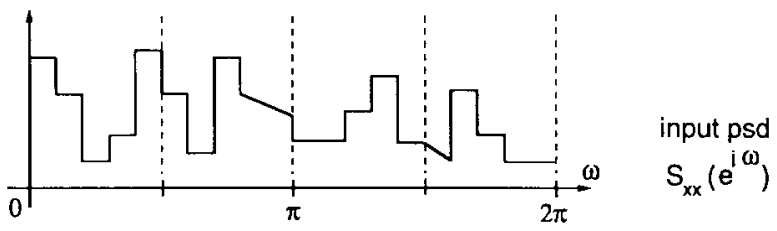

(a)

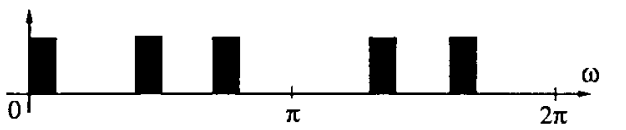

compaction filter $H_{0}\left(e^{i \omega}\right)$

(b)

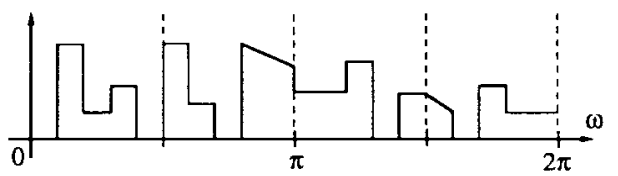

partial psd

$S_{x \alpha}^{(1)}\left(e^{i \omega}\right)$

(c)

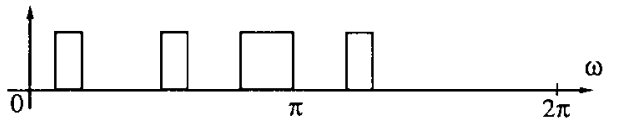

compaction filter $H_{1}\left(e^{j \omega}\right)$

(d)

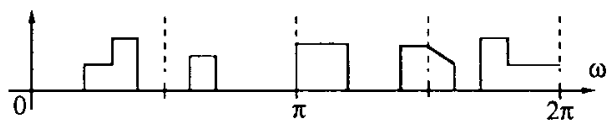

partial psd $S_{x x}^{(2)}\left(e^{i \omega}\right)$

(e)

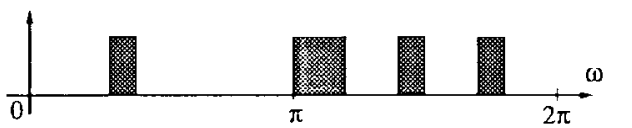

compaction filter $\mathrm{H}_{2}\left(e^{j \omega}\right)$

(f)

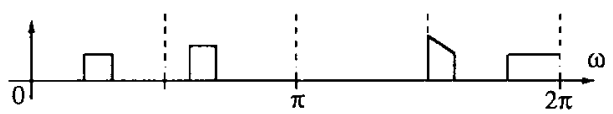

partial pso $S_{x x}^{(3)}\left(e^{j()}\right)$

(g)

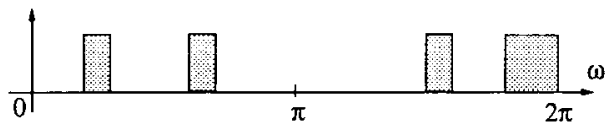

compaction filter $\mathrm{H}_{3}\left(\mathrm{e}^{\mathrm{j} \omega}\right)$

(h)

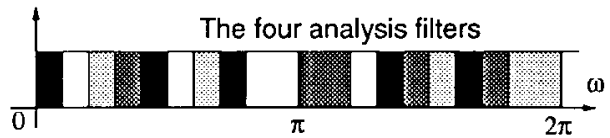

$\begin{array}{llll}\mathrm{H}_{0} & \mathrm{H}_{1} & \mathrm{H}_{2} & \mathrm{H}_{3}\end{array}$

(i)

Fig. 16. Step-by-step description of the construction of a four channel optimum orthonormal filter bank. (a) input psd. (b) Optimum compaction filter. (c)-(h) Partial power spectra and corresponding compaction filters. (i) Summary of the four optimum analysis filters.

is satisfied. As the filters are alias-free, the decimated subband psd $S_{k}\left(e^{j \omega}\right)$ has the value $S_{x x}\left(e^{j \omega_{n_{k}}}\right)$ at the frequency $\left(M \omega_{n_{k}}\right.$ modulo $2 \pi)$. However, since $\omega_{n_{k}}$ are alias-frequencies, the quantity $\hat{\omega}=\left(M \omega_{n_{k}}\right.$ modulo $\left.2 \pi\right)$ is the same for all $k$. It therefore follows from (27) that $S_{0}\left(e^{j \hat{\omega}}\right) \geq S_{1}\left(e^{j \hat{\omega}}\right) \geq \cdots \geq$ $S_{M-1}\left(e^{j \hat{\omega}}\right)$. Since $\omega_{0}$ is arbitrary in $[0,2 \pi / M)$, this statement holds for all $\hat{\omega}$ in $[0,2 \pi)$, proving the majorization property.

$\nabla \nabla \nabla$

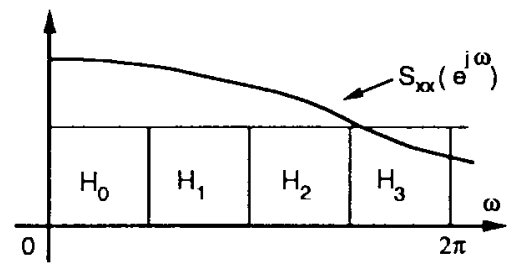

(a)

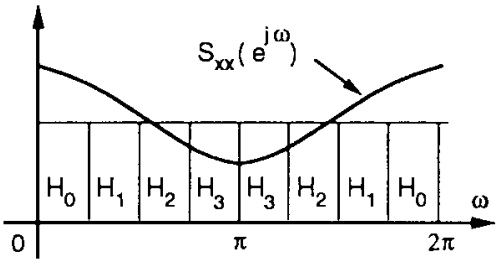

(b)

Fig. 17. Monotone power spectra and optimal orthonormal filter banks. (a) Complex case. (b) Real case (symmetric psd).

Remarks: For the case of monotone nonincreasing input psd, the above optimal design procedure yields the familiar contiguous brickwall stacking. This is demonstrated in Fig. 17 for complex as well as real $x(n)$. Next, if $\left\{H_{k}\left(e^{j \omega}\right)\right\}$ is an optimal orthonormal filter bank for the psd $S_{x x}\left(e^{j \omega}\right)$, then it continues to be optimal for the transformed psd $f\left[S_{x x}\left(e^{j \omega}\right)\right]$, where $f[] \geq$.0 is a nondecreasing function, e.g., for $\sqrt{S_{x x}\left(e^{j \omega}\right)}$. This follows from a similar property of compaction filters (Section V-A).

\section{B. Polyphase Interpretation of Optimality}

We now present a second, algebraic, justification of filter bank optimality by using the polyphase framework. The main advantage of this view is the added insight into the problem. For the next result, recall that the filters are numbered such that the subband signal $v_{0}(n)$ has the highest variance.

Theorem 6: Suppose an $M$-band orthonormal filter bank is optimal for a given input psd $S_{x x}\left(e^{j \omega}\right)$. Then, $H_{0}\left(e^{j \omega}\right)$ is an optimal compaction filter for $S_{x x}\left(e^{j \omega}\right)$.

Proof: The polyphase matrix $\mathbf{E}\left(e^{j \omega}\right)$ of the optimal analysis bank diagonalizes the blocked psd matrix $\mathbf{S}_{B}\left(e^{j \omega}\right)$, as shown in Theorem 1. This diagonalization equation is

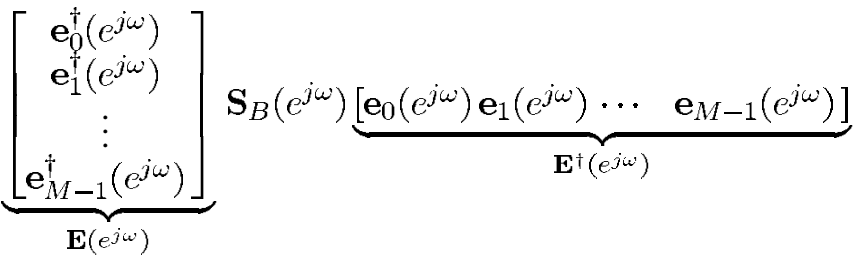

$$
\begin{aligned}
& =\left[\begin{array}{cccc}
S_{0}\left(e^{j \omega}\right) & 0 & \cdots & 0 \\
0 & S_{1}\left(e^{j \omega}\right) & \cdots & 0 \\
\vdots & \vdots & \ddots & \vdots \\
0 & 0 & \cdots & S_{M-1}\left(e^{j \omega}\right)
\end{array}\right] .
\end{aligned}
$$

Since $\mathbf{E}\left(e^{j \omega}\right)$ is unitary, the subband power spectra $S_{k}\left(e^{j \omega}\right)$ are eigenvalues of $\mathbf{S}_{B}\left(e^{j \omega}\right)$. For each frequency $\omega$, the columns of $\mathbf{E}^{\dagger}\left(e^{j \omega}\right)$ are mutually orthogonal unit-norm eigenvectors of $\mathbf{S}_{B}\left(e^{j \omega}\right)$. Since the majorization property is a necessary condition for optimality, it follows that for 
each $\omega, S_{0}\left(e^{j \omega}\right)$ is the maximum eigenvalue of $\mathbf{S}_{B}\left(e^{j \omega}\right)$, and the zeroth column $\mathbf{e}_{0}\left(e^{j \omega}\right)$ of $\mathbf{E}^{\dagger}\left(e^{j \omega}\right)$ is the corresponding eigenvector. By Theorem 5, it follows that $H_{0}\left(e^{j \omega}\right)$ is an optimum compaction filter for $S_{x x}\left(e^{j \omega}\right) . \quad \quad \nabla \nabla \nabla$

Refer again to (28). Due to the majorization property of the optimal filter bank, $S_{1}\left(e^{j \omega}\right)$ is the next largest eigenvalue of $\mathbf{S}_{B}\left(e^{j \omega}\right)$ for each $\omega$. Thus, the vector $\mathbf{e}_{1}\left(e^{j \omega}\right)$ should be such that $\mathbf{e}_{1}^{\dagger}\left(e^{j \omega}\right) \mathbf{S}_{B}\left(e^{j \omega}\right) \mathbf{e}_{1}\left(e^{j \omega}\right)$ is maximized subject to the two orthonormality constraints $\mathbf{e}_{1}^{\dagger}\left(e^{j \omega}\right) \mathbf{e}_{1}\left(e^{j \omega}\right)=1$, and $\mathbf{e}_{1}^{\dagger}\left(e^{j \omega}\right) \mathbf{e}_{0}\left(e^{j \omega}\right)=0$. The elements of $\mathbf{e}_{1}^{\dagger}\left(e^{j \omega}\right)$ are polyphase components of the filter $H_{1}\left(e^{j \omega}\right)$. Therefore, the maximization of $\mathbf{e}_{1}^{\dagger}\left(e^{j \omega}\right) \mathbf{S}_{B}\left(e^{j \omega}\right) \mathbf{e}_{1}\left(e^{j \omega}\right)$ is equivalent to the maximization of the output variance of $H_{1}\left(e^{j \omega}\right)$ with input psd $S_{x x}\left(e^{j \omega}\right)$. Summarizing, $H_{1}\left(e^{j \omega}\right)$ should have maximum output variance under two constraints.

1) $\left|H_{1}\left(e^{j \omega}\right)\right|^{2}$ is $\operatorname{Nyquist}(M)$, i.e., $\mathbf{e}_{1}^{\dagger}\left(e^{j \omega}\right) \mathbf{e}_{1}\left(e^{j \omega}\right)=1$ for all $\omega]$.

2) $\mathbf{e}_{1}^{\dagger}\left(e^{j \omega}\right) \mathbf{e}_{0}\left(e^{j \omega}\right)=0$. Since $H_{0}\left(e^{j \omega}\right)$ is an ideal antialias $(M)$ filter, this orthogonality of the two polyphase vectors is equivalent to the statement that the filters $H_{0}\left(e^{j \omega}\right)$ and $H_{1}\left(e^{j \omega}\right)$ are nonoverlapping:

Lemma 1: Suppose $H_{0}\left(e^{j \omega}\right)$ is designed to be an alias-free $(M)$ filter, i.e., $H_{0}\left(e^{j \omega}\right)$ does not overlap with $H_{0}\left[e^{j(\omega-2 \pi k / M)}\right]$ unless $k$ is a multiple of $M$. Then, $H_{1}\left(e^{j \omega}\right) H_{0}\left(e^{j \omega}\right)=0$ for all $\omega$ if and only if $\mathbf{e}_{1}^{\dagger}\left(e^{j \omega}\right) \mathbf{e}_{0}\left(e^{j \omega}\right)=0$ for all $\omega$. That is, $H_{1}\left(e^{j \omega}\right)$ and $H_{0}\left(e^{j \omega}\right)$ are nonoverlapping if and only if $\mathbf{e}_{1}\left(e^{j \omega}\right)$ is orthogonal to $\mathbf{e}_{0}\left(e^{j \omega}\right)$ for all $\omega$.

Proof: The following relation between the $k$ th filter $H_{k}\left(e^{j \omega}\right)$ and its polyphase vector $\mathbf{e}_{k}\left(e^{j \omega}\right)$ is well-known (see [8, p. 234])

$$
\begin{aligned}
\mathbf{h}_{k}\left(e^{j \omega}\right) & \triangleq\left(\begin{array}{c}
H_{k}\left(e^{j \omega}\right) \\
H_{k}\left[e^{j(\omega-2 \pi / M)}\right] \\
\vdots \\
H_{k}\left\{e^{j[\omega-2 \pi(M-1) / M]}\right\}
\end{array}\right) \\
& =\mathbf{W}^{*} \boldsymbol{\Gamma}\left(e^{j \omega}\right) \mathbf{e}_{k}^{*}\left(e^{j \omega}\right), \quad 0 \leq k \leq M-1
\end{aligned}
$$

where $\mathbf{W}$ is the $M \times M$ DFT matrix, and $\boldsymbol{\Gamma}\left(e^{j \omega}\right)$ is a diagonal matrix with $i$ th diagonal element $e^{-j \omega i}$. Since $\boldsymbol{\Gamma}\left(e^{j \omega}\right)$ and $\mathbf{W}^{*} / \sqrt{M}$ are unitary, it follows that $\mathbf{e}_{1}^{\dagger}\left(e^{j \omega}\right) \mathbf{e}_{0}\left(e^{j \omega}\right)=0$ if and only if $\mathbf{h}_{1}^{\dagger}\left(e^{j \omega}\right) \mathbf{h}_{0}\left(e^{j \omega}\right)=0$. The alias-free property of $H_{0}\left(e^{j \omega}\right)$ means that for any fixed value of $\omega$, there is at most one nonzero element in the column vector $\mathbf{h}_{0}\left(e^{j \omega}\right)$. The condition $\mathbf{h}_{1}^{\dagger}\left(e^{j \omega}\right) \mathbf{h}_{0}\left(e^{j \omega}\right)=0$ [and, hence, the condition $\mathbf{e}_{1}^{\dagger}\left(e^{j \omega}\right) \mathbf{e}_{0}\left(e^{j \omega}\right)=0$ ] is therefore equivalent to the condition $H_{1}\left(e^{j \omega}\right) H_{0}\left(e^{j \omega}\right)=0$.

Having designed $H_{0}\left(e^{j \omega}\right)$ using Theorem $4, H_{1}\left(e^{j \omega}\right)$ should therefore be designed such that

a) $\left|H_{1}\left(e^{j \omega}\right)\right|^{2}$ is $\operatorname{Nyquist}(M)$;

b) $H_{1}\left(e^{j \omega}\right)$ and $H_{0}\left(e^{j \omega}\right)$ are nonoverlapping;

c) the variance at the output of $H_{1}\left(e^{j \omega}\right)$ is maximized for the given input psd $S_{x x}\left(e^{j \omega}\right)$.

For this, we simply peel off the portion of $S_{x x}\left(e^{j \omega}\right)$ falling in the passband of $H_{0}\left(e^{j \omega}\right)$, and then design $H_{1}\left(e^{j \omega}\right)$ to be an optimal compaction filter for the resulting partial psd $S_{x x}^{(1)}\left(e^{j \omega}\right)$. If we design $H_{1}\left(e^{j \omega}\right)$ using the method of Theorem
4, then $H_{1}\left(e^{j \omega}\right)$ is again a two-level alias-free filter that is nonoverlapping with $H_{0}\left(e^{j \omega}\right)$. We can now repeat this argument and identify all the $M$ filters, resulting in the procedure of Section VI-A.

\section{No-Gain Situations}

We conclude this subsection by indicating situations under which there is no coding or compaction gain.

Corollary 2-IncompaCtible Signals: The maximum compaction gain is unity for an input psd $S_{x x}\left(e^{j \omega}\right)$ (for a fixed value of $M)$ if and only if it has the form $S_{x x}\left(e^{j \omega}\right)=$ $S\left(e^{j \omega M}\right)$.

$\nabla \nabla \nabla$

Proof: If $S_{x x}\left(e^{j \omega}\right)=S\left(e^{j \omega M}\right)$, then the psd of the output $v(n)$ in Fig. 10 is $S_{v v}\left(e^{j \omega}\right)=S\left(e^{j \omega}\right)$, as has already been shown in Corollary 1. Therefore, $\sigma_{v}^{2}=\sigma_{x}^{2}$ and the compaction gain $=1$. Conversely, let the maximum compaction gain be unity. Then, the maximum subband variance in the optimal orthonormal SBC is $\sigma_{x_{0}}^{2}=\sigma_{x}^{2}$. However, since $M \sigma_{x}^{2}=\sum_{i} \sigma_{x_{i}}^{2}$ and $\sigma_{x_{0}}^{2} \geq \sigma_{x_{i}}^{2}$ for all $i$, the only possibility is that $\sigma_{x_{i}}^{2}=\sigma_{x}^{2}$ for all $i$. Therefore, the maximum coding gain is unity, and $S_{x x}\left(e^{j \omega}\right)=S\left(e^{j \omega M}\right)$ by Corollary 1. $\quad \nabla \nabla \nabla$

Corollary 3-Unity Compaction Gain and Coding Gain: Let $x(n)$ be a zero mean WSS process with psd $S_{x x}\left(e^{j \omega}\right)$. Then, the following statements are equivalent.

1) $S_{x x}\left(e^{j \omega}\right)$ has the form $S_{x x}\left(e^{j \omega}\right)=S\left(e^{j \omega M}\right)$.

2) Maximum compaction gain $G_{\max }(M)=1$.

3) Any $M$-band orthonormal SBC yields coding gain $=1$.

Proof: The equivalence of statements 1 and 2 comes from the preceding corollary. Statements 1 and 3 are equivalent in view of Corollary 1. Combining these two, it follows that statement 2 and 3 are equivalent as well. [The reason for this is that $(2) \Rightarrow(1) \Rightarrow(3)$. Similarly $(3) \Rightarrow(1) \Rightarrow(2) \cdot] \nabla \nabla \nabla$

\section{Two-Channel Optimal Filter Bank Design}

Although the preceding discussions also hold for the $M=$ 2 case, the simplicity of the two-channel case admits a more direct treatment [14], [18], which also indicates some properties relevant only for the two-channel case. For example, even for finite filter orders, the maximization of coding gain is equivalent to the design of an optimum compaction filter (which is not true for arbitrary $M$ ). The coding gain is $G_{S B C}(2)=\sigma_{x}^{2} / \sqrt{\sigma_{x_{0}}^{2} \sigma_{x_{1}}^{2}}$. Orthonormality implies, in particular, that $\sigma_{x_{0}}^{2}+\sigma_{x_{1}}^{2}=2 \sigma_{x}^{2}$, where $\sigma_{x}^{2}$ is the input variance. Thus

$$
G_{S B C}(2)=\frac{\sigma_{x}^{2}}{\sqrt{\sigma_{x_{0}}^{2}\left(2 \sigma_{x}^{2}-\sigma_{x_{0}}^{2}\right)}} .
$$

Since $\sigma_{x}^{2}$ is fixed, the only variable is $\sigma_{x_{0}}^{2}$, which is the output variance of $H_{0}\left(e^{j \omega}\right)$. To maximize the coding gain, we should minimize the denominator in the preceding expression by choice of $H_{0}\left(e^{j \omega}\right)$ subject to the filter bank orthonormality condition. Orthonormality is equivalent [8] to the Nyquist(2) condition

$$
\left|H_{0}\left(e^{j \omega}\right)\right|^{2}+\left|H_{0}\left(-e^{j \omega}\right)\right|^{2}=2 \quad \text { [Nyquist(2) constraint]. }
$$




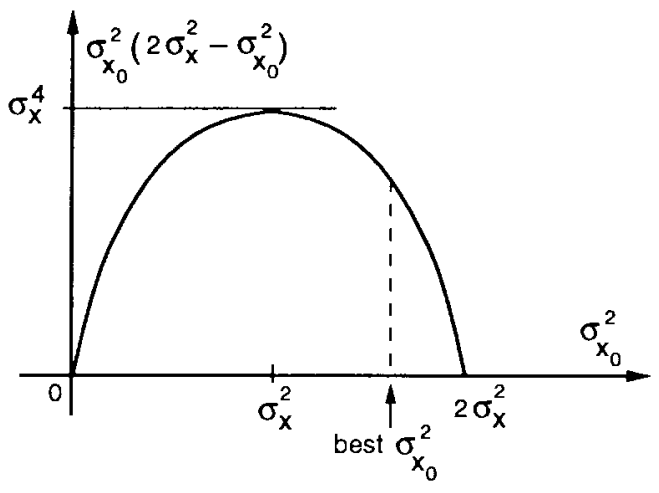

Fig. 18. Pertaining to two-channel optimal orthonormal subband coders.

The quantity $\sigma_{x_{0}}^{2}\left(2 \sigma_{x}^{2}-\sigma_{x_{0}}^{2}\right)$, which occurs in the denominator of (30), is plotted in Fig. 18. (Since $\sigma_{x_{0}}^{2}+\sigma_{x_{1}}^{2}=2 \sigma_{x}^{2}$, the subband variance $\sigma_{x_{0}}^{2}$ has the permissible range $0 \leq \sigma_{x_{0}}^{2} \leq$ $2 \sigma_{x}^{2}$.) To maximize the coding gain, this quantity has to be minimized by choice of $H_{0}\left(e^{j \omega}\right)$ subject to the Nyquist(2) constraint. As the minima in the plot occur at the boundaries, we should make the larger variance $\sigma_{x_{0}}^{2}$ as large as possible. Therefore, we have Theorem 7.

Theorem 7-Two-Channel Optimal Filter Banks: Consider the two-channel orthonormal subband coder (Fig. 1 with $M=$ $2)$. Suppose we design $H_{0}\left(e^{j \omega}\right)$ to be the optimum energy compaction filter for the given input psd $S_{x x}\left(e^{j \omega}\right)$. That is, $H_{0}\left(e^{j \omega}\right)$ is designed such that its output variance $\sigma_{x_{0}}^{2}$ is maximized under the constraint (31). By choosing the other filters $H_{1}\left(e^{j \omega}\right), F_{0}\left(e^{j \omega}\right), F_{1}\left(e^{j \omega}\right)$ in the usual way, we obtain an optimal orthonormal filter bank.

The "usual" way to design the other filters is as follows: First, choose $H_{1}\left(e^{j \omega}\right)$ such that the orthonormality condition $\left[H_{k}\left(e^{j \omega}\right) H_{m}^{*}\left(e^{j \omega}\right)\right]_{\downarrow 2}=\delta(k-m)$ is satisfied. Then, choose the synthesis filters as $F_{k}\left(e^{j \omega}\right)=H_{k}^{*}\left(e^{j \omega}\right)$. For example, if we design the compaction filter $H_{0}\left(e^{j \omega}\right)$ using the method of Theorem 4, then

$$
H_{0}\left(e^{j \omega}\right)= \begin{cases}\sqrt{2} & \text { in the passbands } \\ 0 & \text { in the stopbands. }\end{cases}
$$

Therefore, $H_{1}\left(e^{j \omega}\right)$ is obtained by interchanging the passband and stopband, or equivalently, $H_{1}\left(e^{j \omega}\right)=\sqrt{2}-H_{0}\left(e^{j \omega}\right)$.

Finite-Order Filters: For arbitrary $M$, the connection between energy compaction and optimal coding gain (Section VI-A) holds only when there is no constraint on the filter orders. However, for the two-channel case, the results are valid even for finite-order filters (FIR or IIR), as seen from the arguments which lead to Theorem 7 . The remaining three filters $H_{1}\left(e^{j \omega}\right), F_{0}\left(e^{j \omega}\right)$, and $F_{1}\left(e^{j \omega}\right)$ are determined in terms of $H_{0}\left(e^{j \omega}\right)$ by standard relations for two-channel orthonormal filter banks [8], namely, $H_{1}(z)=-z^{-N} \tilde{H}_{0}(-z)$ for odd $N, F_{0}(z)=\tilde{H}_{0}(z)$, and $F_{1}(z)=\tilde{H}_{1}(z)$. Here, $\tilde{F}(z)$ stands for $F^{*}\left(1 / z^{*}\right)$.

\section{PROPERTIES OF OPTIMUM COMPACTION AND CODING GAINS}

In Fig. 10, the compaction gain is defined as the ratio $G_{\text {comp }}(M)=\sigma_{v}^{2} / \sigma_{x}^{2}$ (equivalently, $\sigma_{y}^{2} / \sigma_{x}^{2}$ since $\sigma_{v}^{2}=\sigma_{y}^{2}$ ).
This gain depends on the input psd, the integer $M$, and the filter $H\left(e^{j \omega}\right)$. For example, if the psd $S_{x x}\left(e^{j \omega}\right)$ is bandlimited to $[-\pi / 2, \pi / 2)$ and the filter is chosen to have passband for $\pi / 2 \leq|\omega|<\pi$, then the output is zero, and $G_{c o m p}(2)=0$. Thus, the uninteresting lower bound on $G_{c o m p}(M)$ is zero. It is more important to study the properties of the maximum compaction gain $G_{\max }(M)$, which arises when $H\left(e^{j \omega}\right)$ is chosen as the optimal compaction filter for $S_{x x}\left(e^{j \omega}\right)$. Evidently, $G_{\max }(M) \geq 1$ because the choice $H\left(e^{j \omega}\right)=1$ satisfies the Nyquist constraint and yields $G_{c o m p}(M)=1$. Next, since $\left|H\left(e^{j \omega}\right)\right|^{2} \leq M$ (Section V), $\sigma_{y}^{2}=\int_{0}^{2 \pi} S_{x x}\left(e^{j \omega}\right)\left|H\left(e^{j \omega}\right)\right|^{2} d \omega / 2 \pi \leq$ $M \int_{0}^{2 \pi} S_{x x}\left(e^{j \omega}\right) d \omega / 2 \pi=M \sigma_{x}^{2}$. Thus

$$
1 \leq G_{\max }(M) \leq M \text {. }
$$

Therefore, there is an input-independent upper bound $M$. By contrast, the coding gain of an orthonormal subband coder [the $A M / G M$ ratio (3)] has no such bound [e.g., choose $S_{x x}\left(e^{j \omega}\right)$ such that a subband has arbitrarily small variance]. We already know (Corollary 2) that the lower bound $G_{\max }(M)=1$ is attained iff $S_{x x}\left(e^{j \omega}\right)$ has the form $S\left(e^{j \omega M}\right)$. The conditions that yield the upper bound $G_{\max }(M)=M$ are given next.

Theorem 8-Signal with Best Compaction Gain: For fixed $M$, the maximum compaction gain $G_{\max }(M)$ attains the upper bound $G_{\max }(M)=M$ if and only if the support $\mathcal{S}$ of the input psd $S_{x x}\left(e^{j \omega}\right)$ is an aliasfree $(M)$ support (defined in Section I-A).

Proof: First, assume that $S_{x x}\left(e^{j \omega}\right)$ is aliasfree $(M)$ and construct $H\left(e^{j \omega}\right)$ according to Theorem 4 . The set $\left\{S_{x x}\left(e^{j \omega_{k}}\right)\right\}$ described in that theorem will have at most one nonzero element (by aliasfree property). Therefore, the resulting $H\left(e^{j \omega}\right)$ will be such that its passband includes the support $\mathcal{S}$ of $S_{x x}\left(e^{j \omega}\right)$. Since $\left|H\left(e^{j \omega}\right)\right|^{2}=M$ in the passband, we get

$$
\begin{aligned}
\sigma_{y}^{2} & =\int_{0}^{2 \pi} S_{x x}\left(e^{j \omega}\right)\left|H\left(e^{j \omega}\right)\right|^{2} d \omega / 2 \pi \\
& =\int_{\mathcal{S}} S_{x x}\left(e^{j \omega}\right)\left|H\left(e^{j \omega}\right)\right|^{2} d \omega / 2 \pi \\
& =M \int_{0}^{2 \pi} S_{x x}\left(e^{j \omega}\right) d \omega / 2 \pi=M \sigma_{x}^{2} .
\end{aligned}
$$

This shows that $G_{\max }(M)=\sigma_{y}^{2} / \sigma_{x}^{2}=M$. Conversely, suppose that $S_{x x}\left(e^{j \omega}\right)$ is such that $G_{\max }(M)=M$. Therefore, any optimal filter yields $\sigma_{y}^{2}=M \sigma_{x}^{2}$, that is, $\int_{0}^{2 \pi} S_{x x}\left(e^{j \omega}\right)\left|H\left(e^{j \omega}\right)\right|^{2} d \omega / 2 \pi=M \int_{0}^{2 \pi} S_{x x}\left(e^{j \omega}\right) d \omega / 2 \pi$, or

$$
\int_{0}^{2 \pi}\left[M-\left|H\left(e^{j \omega}\right)\right|^{2}\right] S_{x x}\left(e^{j \omega}\right) d \omega / 2 \pi=0 .
$$

Since $\left|H\left(e^{j \omega}\right)\right|^{2} \leq M$, we have $\left(M-\left|H\left(e^{j \omega}\right)\right|^{2}\right) \geq 0$, and the integrand above is nonnegative. Therefore, we conclude that $\left(M-\left|H\left(e^{j \omega}\right)\right|^{2}\right) S_{x x}\left(e^{j \omega}\right)=0$ for all $\omega$. That is, whenever $S_{x x}\left(e^{j \omega}\right) \neq 0$, we have to have $\left|H\left(e^{j \omega}\right)\right|^{2}=$ $M$. This statement holds for any optimal solution, including the aliasfree $(M)$ filter of Theorem 4. Thus, the support of $S_{x x}\left(e^{j \omega}\right)$ is contained within the passband of the optimal filter $H\left(e^{j \omega}\right)$, showing that $S_{x x}\left(e^{j \omega}\right)$ has aliasfree $(M)$ support. 


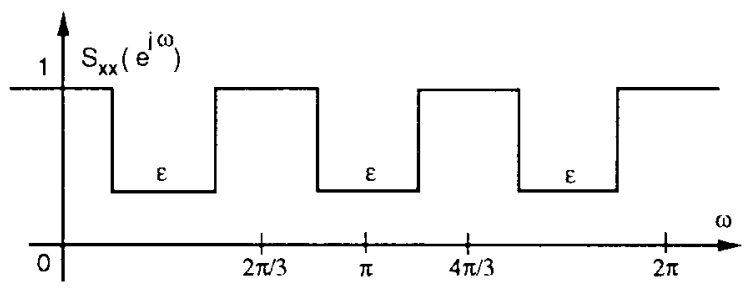

(a)

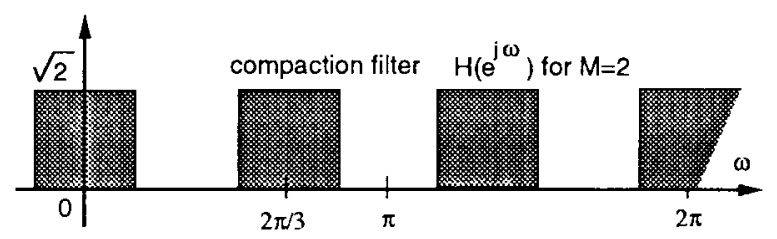

(b)

Fig. 19. Example where compaction gain decreases with increasing $M$. (a) Input psd. (b) Compaction filter for $M=2$. There is no compaction gain for $M=3$.

\section{A. Optimum Compaction Gain as a Function of $M$}

The optimum compaction gain $G_{\max }(M)$ is not necessarily a monotone function of $M$. Thus, if the psd has the form $S_{x x}\left(e^{j \omega}\right)=S\left(e^{j \omega M}\right)$, then $G_{\max }(M)=1$, but we have $G_{\max }(M-1)>1$ unless $S_{x x}\left(e^{j \omega}\right)$ also has the form $S_{1}\left[e^{j \omega(M-1)}\right]$ (see Corollary 2). For example in Fig. 19(a), where $\epsilon<1, G_{\max }(2)=2 /(1+\epsilon)>1$, but $G_{\max }(3)=$ $1<G_{\max }(2)$. Notice that for any input psd, the compaction gain does satisfy $G_{\max }(k M) \geq G_{\max }(M)$ for any choice of integer $k>1$. The reason for this is that if a filter $\left|H\left(e^{j \omega}\right)\right|^{2}$ is Nyquist $(M)$, it is also Nyquist $(k M)$. Therefore, $H\left(e^{j \omega}\right)$ remains a valid compaction filter when $M$ is replaced with $k M$. We also have the following restricted monotone property.

Theorem 9-Compaction Gain for Monotone psd: Let the psd $S_{x x}\left(e^{j \omega}\right)$ be nonincreasing in $0 \leq \omega<2 \pi$, e.g., as in Fig. 17(a). Then, the compaction gain $G_{\max }(M)$ is a nondecreasing function of $M$. The same is true also for real signals with monotone psd in $0 \leq \omega \leq \pi$, e.g., Fig. 17(b). $\diamond$

Proof: If the psd is nonincreasing in $0 \leq \omega<2 \pi$, the optimal compaction filter can be taken to be lowpass (Section V-A). Fig. 20 shows sketches of the optimal filter $\left|H\left(e^{j \omega}\right)\right|$ for the cases $M$ and $M+1$. The output variances are

$$
\begin{aligned}
\sigma_{y}^{2}(M) & =M \int_{0}^{2 \pi / M} S_{x x}\left(e^{j \omega}\right) d \omega / 2 \pi \\
\sigma_{y}^{2}(M+1) & =(M+1) \int_{0}^{2 \pi /(M+1)} S_{x x}\left(e^{j \omega}\right) d \omega / 2 \pi
\end{aligned}
$$

Since $G_{\max }(M)=\sigma_{y}^{2}(M) / \sigma_{x}^{2}$ and $G_{\max }(M+1)=\sigma_{y}^{2}(M+$ 1) $/ \sigma_{x}^{2}$, we only have to show that $\sigma_{y}^{2}(M) \leq \sigma_{y}^{2}(M+1)$. Imagine that the region $0 \leq \omega<2 \pi / M$ is divided uniformly into $M+1$ consecutive pieces of length $2 \pi / M(M+1)$ each. In view of the monotone property of $S_{x x}\left(e^{j \omega}\right)$, we have

$$
\int_{2 \pi /(M+1)}^{2 \pi / M} S_{x x}\left(e^{j \omega}\right) d \omega \leq \frac{1}{M+1} \int_{0}^{2 \pi / M} S_{x x}\left(e^{j \omega}\right) d \omega
$$

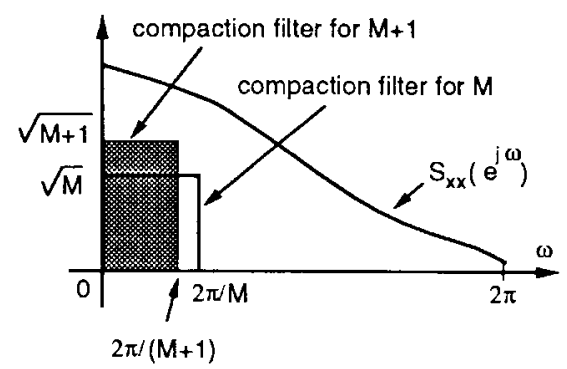

Fig. 20. Pertaining to the fact that the compaction gain is monotone for monotone psd.

$$
\begin{aligned}
\text { Now } & \begin{aligned}
\int_{0}^{2 \pi / M} S_{x x}\left(e^{j \omega}\right) d \omega= & \int_{0}^{2 \pi /(M+1)} S_{x x}\left(e^{j \omega}\right) d \omega \\
& +\int_{2 \pi /(M+1)}^{2 \pi / M} S_{x x}\left(e^{j \omega}\right) d \omega \\
\leq & \int_{0}^{2 \pi /(M+1)} S_{x x}\left(e^{j \omega}\right) d \omega \\
& +\frac{1}{M+1} \int_{0}^{2 \pi / M} S_{x x}\left(e^{j \omega}\right) d \omega
\end{aligned}
\end{aligned}
$$

by using (37). Rearranging this, we get

$$
\begin{aligned}
& \left(1-\frac{1}{M+1}\right) \int_{0}^{2 \pi / M} S_{x x}\left(e^{j \omega}\right) d \omega \\
& \quad \leq \int_{0}^{2 \pi /(M+1)} S_{x x}\left(e^{j \omega}\right) d \omega .
\end{aligned}
$$

Simplifying this and using (36), we have $\sigma_{y}^{2}(M) \leq \sigma_{y}^{2}(M+1)$ so that $G_{\max }(M+1) \geq G_{\max }(M)$ indeed. For real signals [psd as in Fig. 17(b)], we use integrals like $\int_{0}^{\pi} S_{x x}\left(e^{j \omega}\right) d \omega / \pi$ and make similar arguments.

$\nabla \nabla \nabla$

Asymptotic Compaction Gain: Note that the output variance of the compaction filter is

$$
\begin{aligned}
\sigma_{y}^{2} & =\int_{0}^{2 \pi} S_{x x}\left(e^{j \omega}\right)\left|H\left(e^{j \omega}\right)\right|^{2} d \omega / 2 \pi \\
& \leq S_{\max } \int_{0}^{2 \pi}\left|H\left(e^{j \omega}\right)\right|^{2} d \omega / 2 \pi=S_{\max }
\end{aligned}
$$

where $S_{\max }$ is the peak value of $S_{x x}\left(e^{j \omega}\right)$. Here, we have used the unity energy constraint $\int\left|H\left(e^{j \omega}\right)\right|^{2} d \omega / 2 \pi=1$, which follows from the Nyquist condition (16). Thus, for any input psd, the maximum possible compaction gain with no constraint on $M$ is

$$
\mathcal{G}=\frac{S_{\max }}{\sigma_{x}^{2}}=\frac{S_{\max }}{\int_{0}^{2 \pi} S_{x x}\left(e^{j \omega}\right) d \omega / 2 \pi}=\frac{\text { peak psd }}{\text { average psd }} .
$$

If the filter $H\left(e^{j \omega}\right)$ is restricted only by the unit energy constraint rather than the stronger Nyquist constraint, we can approach the above bound trivially by using a unit-energy bandpass filter with arbitrarily narrow passband (Fig. 21). Therefore, the only nontrivial aspect of the energy compaction concept comes from the imposition of the $\operatorname{Nyquist}(M)$ constraint. Since the filter in Fig. 21 satisfies this constraint, it is a valid compaction filter, leading to the conclusion 


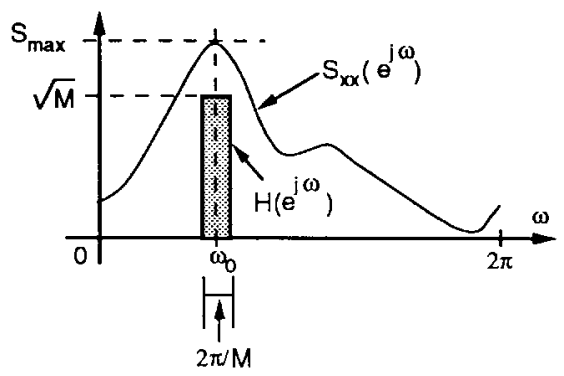

Fig. 21. Attaining maximum compaction gain asymptotically with bandpass filters.

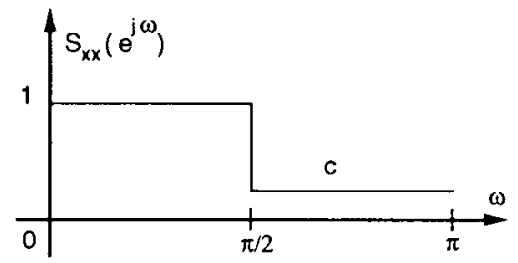

(a)

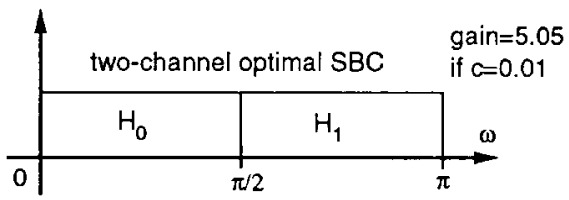

(b)

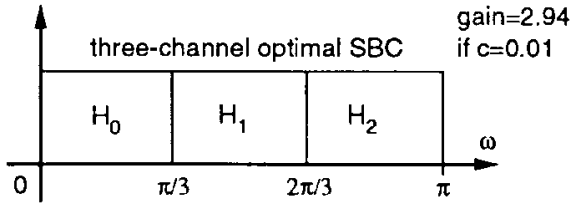

(c)

Fig. 22. Demonstrating that the optimal coding gain may not be monotone in $M$, even for monotone psd. (a) Input psd. (b) and (c) Optimal orthonormal SBC for $M=2$ and 3 .

$G_{\max }(\infty)=S_{\max } / \sigma_{x}^{2}$. A consequence of this result is that $G_{\max }(M)=1$ for all $M$ if and only if $x(n)$ is white because we have $S_{\max }=\sigma_{x}^{2}$ only when $S_{x x}\left(e^{j \omega}\right)$ is flat.

\section{B. Optimum Subband Coding Gain as Function of $M$}

Assume that the psd is nonincreasing in $\omega$ so that the maximum compaction gain is nondecreasing in $M$. With $G_{S B C}(M)$ denoting the coding gain of the optimal orthonormal subband coder, it does not still follow that $G_{S B C}(M)$ is nondecreasing! For example, consider the monotone psd shown in Fig. 22(a). The optimal orthonormal filter bank in this case is the contiguously stacked brickwall filter bank (see the end of Section VI-A), as indicated in the figure for $M=2$ and $M=3$. With $c=0.01$, we can verify that $G_{S B C}(2)=5.05$ and $G_{S B C}(3)=2.94<G_{S B C}(2)$. In fact, since the psd is a piecewise constant with the edges aligned along the alias-free zones of the two-band subband coder, $G_{S B C}(M) \leq G_{S B C}(2)$ for any $M$ (Section IX).

For optimal orthonormal SBC, we have $G_{S B C}(k M) \geq$ $G_{S B C}(M)$. This is because given an optimal $M$-band solution with ideal aliasfree $(M)$ filters, imagine we split each band further into $k$ uniform regions using ideal filters. Then, the coding gain (the $A M / G M$ formula) cannot decrease, which proves the point.

\section{RELATIVE BEHAVIORS OF KLT, LPC, AND SBC}

We now point out some basic differences in the behaviors of optimal transform coders, subband coders, and linear predictors. A $k$ th-order linear predictor for the (zero-mean) process $x(n)$ has prediction error $e(n)=x(n)-\sum_{i=1}^{k} a_{i} x(n-i)$, where the coefficients $a_{i}$ are the predictor coefficients. If these coefficients are chosen to minimize $E|e(n)|^{2}$, we have an optimal predictor. Let $\mathcal{E}_{k}$ denote this minimized mean square prediction error for the $k$ th-order predictor. With $\sigma_{x}^{2}$ denoting the variance of $x(n)$, the prediction gain is

$$
G_{L P C}(k)=\frac{\sigma_{x}^{2}}{\mathcal{E}_{k}} .
$$

It is known [3], [26], [27] that as the prediction order $k \rightarrow$ $\infty$, we have $\mathcal{E}_{\infty}=\exp \left(\int_{0}^{2 \pi} \operatorname{Ln} S_{x x}\left(e^{j \omega}\right) d \omega / 2 \pi\right)$ (under a few mild technical conditions). The corresponding asymptotic prediction gain is defined as

$$
\begin{aligned}
G_{L P C}(\infty) & =\frac{\sigma_{x}^{2}}{\exp \left[\int_{0}^{2 \pi} \operatorname{Ln} S_{x x}\left(e^{j \omega}\right) d \omega / 2 \pi\right]} \\
& =\frac{\int_{0}^{2 \pi} S_{x x}\left(e^{j \omega}\right) d \omega / 2 \pi}{\exp \left[\int_{0}^{2 \pi} \operatorname{Ln} S_{x x}\left(e^{j \omega}\right) d \omega / 2 \pi\right]} .
\end{aligned}
$$

This is also known [3], [8] to be the coding gain of the contiguously stacked brickwall subband coder (Fig. 2) as the number of channels $M \rightarrow \infty$. Furthermore, it is known [3] that this ratio is also the coding gain of the optimal orthonormal transform coder $(K L T)$ as the block size $M \rightarrow \infty$. Finally, the differential PCM (or DPCM) system [3] has a coding gain that approaches the ratio $G_{L P C}(\infty)$ as the order of the optimal predictor in its feedback loop grows indefinitely. In fact, when $x(n)$ is a Gaussian process, $G_{L P C}(\infty)$ is the rate-distortiontheoretic bound on the coding gain (for any scheme). ${ }^{1}$ Thus, the three data compression systems, namely, the SBC, KLT, and DPCM, approach the rate distortion bound asymptotically. The reciprocal quantity $\gamma_{x}^{2}=1 / G_{L P C}(\infty)$ is usually called the spectral flatness measure. We have $0<\gamma_{x}^{2} \leq 1$, with $\gamma_{x}^{2}=1$ if and only if $x(n)$ is white.

While the behaviors of SBC, KLT, and DPCM are similar asymptotically, there are subtle differences for finite $M$. Let

$G_{K L T}(M)$ coding gain (under optimal bit allocation) of the $M$-band optimal orthonormal transform coder (KLT);

$G_{S B C}(M) \quad$ coding gain of the $M$-band optimal orthonormal subband coder;

$G_{L P C}(M)$ prediction gain of the $M$ th-order optimal predictor.

\footnotetext{
${ }^{1}$ Strictly speaking, this holds only in the so-called high-bit rate or "small
} error" case; see [3] and [26]. 
1) $G_{K L T}(M)$ is a nondecreasing function of $M$. Moreover, if two successive values of the KLT gain are identical, a degenerate situation is implied. More specifically, $G_{K L T}(M)=G_{K L T}(M+1)$ if and only if the autocorrelation $R_{x x}(k)$ of the process $x(n)$ satisfies $R_{x x}(k)=$ 0 for $1 \leq k \leq M$. This means in particular that $G_{K L T}(k)=1$ for $1 \leq k \leq M+1$ and $G_{L P C}(k)=1$ for $1 \leq k \leq M$. Thus, $G_{K L T}(M)$ cannot saturate for finite $M$ unless $x(n)$ is white. Proofs can be found in [8, Appendix C].

2) $G_{K L T}(M+1) \leq G_{L P C}(M)$. Moreover, if $G_{K L T}(M+$ $1)=G_{L P C}(M)$, then we again have the same degenerate situation $R_{x x}(k)=0$ for $1 \leq k \leq M$. These results are proved by a slight modification of the proofs of the results in the preceding paragraph.

3) $G_{L P C}(M)$ is also a nondecreasing function of $M$ [27]. However, the situation $G_{L P C}(M)=G_{L P C}(M+1)$ does not imply any degeneracy as in the KLT case. In fact, unlike the KLT gain, $G_{L P C}(M)$ can saturate for finite $M$ (as for autoregressive processes).

4) $G_{S B C}(M)$, which is the coding gain of the optimal orthonormal $M$-band subband coder, is not necessarily monotone in $M$ (Section VII-B). Moreover, it can attain the prediction bound for finite $M$ for a certain class of processes (Section IX). However, it cannot saturate unless $x(n)$ is white. That is, we cannot have $G_{S B C}(k)=G_{S B C}(\infty)$ for all $k \geq M$ unless $x(n)$ is white. We will not prove this here, but request verification of a closely related statement: Consider a brickwall subband coder with contiguous stacking (e.g., Fig. 2). If $G_{S B C}(M)=G_{S B C}(N)=G_{S B C}(\infty)$ for a relatively prime pair of integers $M, N>1$, then it turns out that $x(n)$ is white. In particular, therefore, if $G_{S B C}(M)=G_{S B C}(M+1)=G_{S B C}(\infty)$ (which is implied by saturation), then $x(n)$ is white.

Example: Fig. 23 shows two examples of input psd for $M=2$. The prediction gain $G_{L P C}(1), K L T$ gain $G_{K L T}(2)$, and subband coder gain $G_{S B C}(2)$ (contiguous filter stacking) are, for Case 1, $G_{L P C}(1)=1.638, G_{K L T}(2)=1.28$, and $G_{S B C}(2)=5.05$ so that $G_{S B C}(2)>G_{K L T}(2)$. However, for Case $2, G_{L P C}(1)=1.0078, G_{K L T}(2)=1.004$, and $G_{S B C}(2)=1$. Thus, if the subband coder filters are not optimized, KLT might out perform SBC, depending on the nature of the input psd.

KLT and Energy Compaction: The relation between energy compaction and coding gain was shown in Section VI, assuming the analysis filters to be of unrestricted order. For the special case of the KLT, where the filters have order $\leq M-1$, this relation happens to be still true. (If $M \leq N<\infty$, then there is no simple relation between the optimal coding gain and optimal energy compaction). For orthonormal transform coders, the polyphase matrix $\mathbf{E}\left(e^{j \omega}\right)$ is a constant unitary matrix. The solution that maximizes the coding gain is the KLT, which decorrelates $v_{k}(n)$ and $v_{m}(n)$ for each $n$. The result is such that $v_{0}(n)$ has the maximum variance [equal to the maximum eigenvalue of the correlation matrix of the blocked process $\left.\mathbf{x}_{B}(n)\right]$. Therefore, the zeroth subband filter $H_{0}(z)$ is the optimal compaction filter, under the FIR
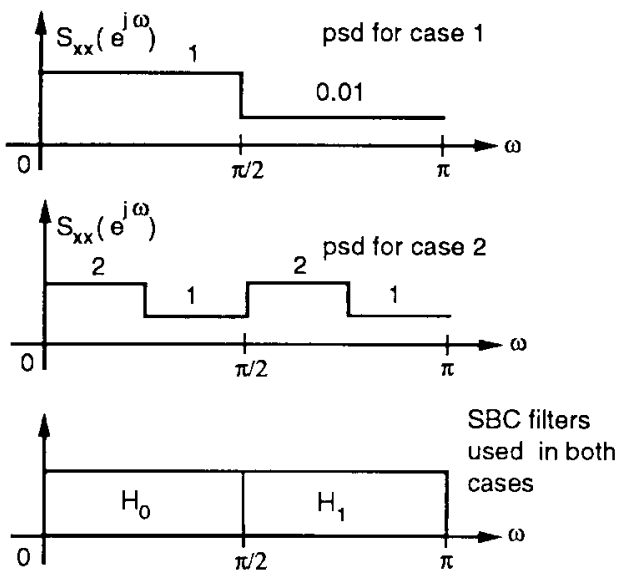

Fig. 23. Two cases of input power spectrum for comparison of KLT with SBC.

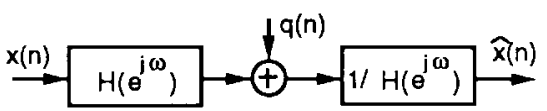

Fig. 24. One-channel biorthogonal filter bank.

constraint with order $\leq M-1$. The next subband signal $v_{1}(n)$ has variance equal to the next largest eigenvalue. This corresponds to a compaction filter, constrained to be of order $\leq M-1$ and orthogonal to the preceding one, i.e., the impulse responses of $H_{0}(z)$ and $H_{1}(z)$ are the components of the first two orthogonal eigenvectors of the correlation matrix of $\mathrm{x}_{B}(n)$.

\section{CONCLUding ReMARKS: When IS ORTHONORMAL SBC AS GOOD AS BIORTHOGONAL?}

A biorthogonal filter bank satisfies (1a) but not necessarily (1b). The general expression for the coding gain in this case is derived in [22] by using the standard noise model of Section I with the further assumption that the noise sources be white and uncorrelated. For fixed number of subbands $M$, biorthogonal filter banks can, in general, provide better coding gain than orthonormal filter banks (except for the special case of transform coders; see the Appendix). This can be demonstrated easily by taking the case $M=1$. Then, the "subband coder" reduces to the quantizer with a prefilter $H\left(e^{j \omega}\right)$ and a postfilter $1 / H\left(e^{j \omega}\right)$, as shown in Fig. 24. In the orthonormal case, $H\left(e^{j \omega}\right)$ is allpass, and there is no coding gain for any input psd $S_{x x}\left(e^{j \omega}\right)$. If we lift the orthonormality (or allpass) requirement, then the best coding gain (under the standard noise model assumption of Section I plus the added requirement that the noise be white) is obtained by the halfwhitening solution [3] given by $H\left(e^{j \omega}\right)=1 /\left[S_{x x}\left(e^{j \omega}\right)\right]^{1 / 4}$. In fact, one corollary of the main result in [22] is that the coding gain of an optimal orthonormal filter bank can almost always be improved by using a half-whitening prefilter $H\left(e^{j \omega}\right)$ and a post filter $1 / H\left(e^{j \omega}\right)$ around that filter bank.

We will now show that if the input psd $S_{x x}\left(e^{j \omega}\right)$ has a certain structure (which depends on $M$ ), then no biorthogonal filter bank can do better that the best orthonormal filter bank. To motivate this, consider Fig. 25(a), where we have 


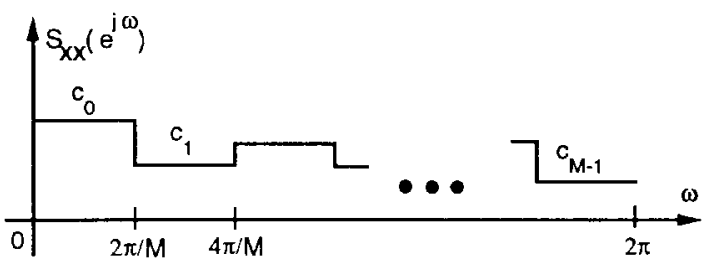

(a)

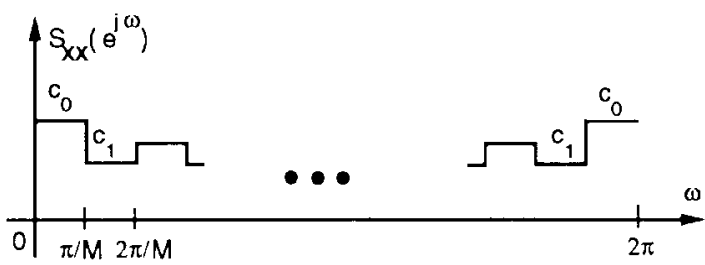

(b)

Fig. 25. Piecewise constant power spectra. (a) Example of a complex process. (b) Example of a real process where the psd is symmetric.

a psd that is piecewise constant. The regions of constancy are contiguous frequency segments of length $2 \pi / M$. Fig. 25(b) shows a different example where the regions of constancy are symmetric with respect to zero frequency (as in the case of real input). If we use brickwall filter banks with contiguous stacking as demonstrated in Fig. 2(a) and (b), respectively, then the subband signal $x_{k}(n)$ has a constant psd in the passband. Since the filters are ideal aliasfree $(M)$ filters, the decimated signals $v_{k}(n)$ have constant psd everywhere. Thus, the set of decimated subband signals $\left\{v_{k}(n)\right\}$ satisfy the decorrelation as well as majorization properties. The filter bank is therefore an optimal orthonormal filter bank (Theorem 3). We will now see that this orthonormal filter bank is optimal even over the class of biorthogonal filter banks. That is, no $M$-channel biorthogonal system can provide a better coding gain. For this, first note that since the psd $S_{x x}\left(e^{j \omega}\right)$ has the constant value $\sigma_{x_{i}}^{2}$ in the $i$ th subband, we can rewrite

$$
\begin{aligned}
\exp \int_{0}^{2 \pi} \operatorname{Ln}\left[S_{x x}\left(e^{j \omega}\right)\right] d \omega / 2 \pi & =\exp \frac{1}{M} \sum_{i=0}^{M-1} \operatorname{Ln} \sigma_{x_{i}}^{2} \\
& =\left(\prod_{i} \sigma_{x_{i}}^{2}\right)^{1 / M}
\end{aligned}
$$

Thus, the coding gain $\sigma_{x}^{2} /\left(\prod_{i} \sigma_{x_{i}}^{2}\right)^{1 / M}$ is equal to (42). Suppose there existed a biorthogonal filter bank with better coding gain. Since the subband coder gain depends only on $S_{x x}\left(e^{j \omega}\right)$ and not on whether $x(n)$ is Gaussian or not, we see that there will exist a Gaussian process for which the biorthogonal coding gain is larger than (42), violating the upper bound given by rate distortion theory. Therefore, the brickwall (orthonormal) SBC achieves best coding gain. We cannot do better by using biorthogonal filter banks.

How about the converse? Imagine that we have found the optimal orthonormal filter bank $\left\{H_{k}\left(e^{j \omega}\right)\right\}$, e.g., by using the method in Section VI-A, and that no biorthogonal filter bank can yield a better gain. The passbands of the filters $\left\{H_{k}\left(e^{j \omega}\right)\right\}$ define $M$ aliasfree $(M)$ zones $\mathcal{R}_{k}$ as shown in Section VI-A. If the portion of $S_{x x}\left(e^{j \omega}\right)$ falling under an aliasfree $(M)$ zone
$\mathcal{R}_{i}$ is not a constant, then the psd of the decimated subband $v_{i}(n)$ is not a constant, and we can insert a half-whitening filter $G_{i}\left(e^{j \omega}\right)$ and reduce the quantization error. This results in an effective biorthogonal filter bank, whose $i$ th analysis filter is $H_{i}(z) G_{i}\left(z^{M}\right)$, which improves the coding gain, violating the starting assumption. Therefore, we have proved the following theorm.

Theorem 10-Orthonormal SBC versus Biorthgonal SBC: Assume that the number of channels $M$ is fixed. Let the quantizers be modeled with the standard noise model of Section I-A with the additional assumption that the noise sources are white and pairwise uncorrelated. Then, the following two statements are equivalent.

1) The optimal orthonormal subband coding gain is at least as large as that of any biorthogonal system.

2) The frequency region $[0,2 \pi)$ can be partitioned into $M$ aliasfree $(M)$ regions such that the input psd $S_{x x}\left(e^{j \omega}\right)$ is constant in each zone. That is, the input psd is a piecewise constant with respect to an aliasfree $(M)$ partition.

\section{APPENDIX}

\section{GENERALITY OF ORTHOGONAL TRANSFORM CODERS}

We now show that for the transform coder case, where $\mathbf{E}\left(e^{j \omega}\right)$ is a constant nonsingular matrix $\mathbf{T}$, orthonormality is not a loss of generality, unlike subband coders. More precisely, we have the following theorem.

Theorem 11: In Fig. 1(b), assume that $\mathbf{E}\left(e^{j \omega}\right)$ is a constant nonsingular matrix denoted as $\mathbf{T}$. Assume the standard noise model (Section I-A), and, in addition, that the quantizer errors are (zero-mean and) uncorrelated, that is, $E\left[q_{k}(n) q_{m}^{*}(n)\right]=0$ for $k \neq m$ and for all $n$. Then, the transformation $\mathbf{T}$ that minimizes the reconstruction error variance with optimal bit allocation is such that $\mathbf{T} \mathbf{T}^{\dagger}$ is diagonal. We can assume the optimal $\mathbf{T}$ to be unitary, that is, $\mathbf{T T}+=\mathbf{I}$.

Proof: Denote $\mathbf{S}=\mathbf{T}^{-1}$. The signal reconstruction error $\mathbf{e}(n) \triangleq \widehat{\mathbf{x}}(n)-\mathbf{x}(n)$ and the quantizer error $\mathbf{q}(n) \triangleq \widehat{\mathbf{y}}(n)-$ $\mathbf{y}(n)$ are related as $\mathbf{e}(n)=\mathbf{S q}(n)$. The reconstruction error variance is

$$
\begin{aligned}
\mathcal{E} & =E\left[\mathbf{e}^{\dagger}(n) \mathbf{e}(n)\right]=\operatorname{Tr}\left\{E\left[\mathbf{e}(n) \mathbf{e}^{\dagger}(n)\right]\right\} \\
& =\operatorname{Tr}\left\{\mathbf{S} E\left[\mathbf{q}(n) \mathbf{q}^{\dagger}(n)\right] \mathbf{S}^{\dagger}\right\}=\operatorname{Tr}\left\{\mathbf{S}^{\dagger} \mathbf{S} E\left[\mathbf{q}(n) \mathbf{q}^{\dagger}(n)\right]\right\}
\end{aligned}
$$

using $\operatorname{Tr}(\mathbf{A B})=\operatorname{Tr}(\mathbf{B A})$. Since $E\left[q_{k}(n) q_{m}^{*}(n)\right]=0$ for $k \neq m$, the matrix $E\left[\mathbf{q}(n) \mathbf{q}^{\dagger}(n)\right]$ is a diagonal matrix with diagonal elements $\sigma_{q_{i}}^{2}$. Thus, the matrix $\mathbf{S}^{\dagger} \mathbf{S} E\left[\mathbf{q}(n) \mathbf{q}^{\dagger}(n)\right]$ has the diagonal elements $\sigma_{q_{i}}^{2}\left\|\mathbf{s}_{i}\right\|^{2}$, where $\mathbf{s}_{i}$ are the columns of S. This yields

$$
\begin{aligned}
\mathcal{E} & =\sum_{i=0}^{M-1} \sigma_{q_{i}}^{2}\left\|\mathbf{s}_{i}\right\|^{2}=c \sum_{i=0}^{M-1} 2^{-2 b_{i}} \sigma_{y_{i}}^{2}\left\|\mathbf{s}_{i}\right\|^{2} \\
& \geq c M 2^{-2 b}\left(\prod_{i=0}^{M-1} \sigma_{y_{i}}^{2}\left\|\mathbf{s}_{i}\right\|^{2}\right)^{1 / M} .
\end{aligned}
$$

This follows by using the $A M-G M$ inequality along with the average bit constraint $b=\sum_{i=0}^{M-1} b_{i} / M$. Define the matrices 
$\mathbf{R}_{x x}=E\left[\mathbf{x}(n) \mathbf{x}^{\dagger}(n)\right]$ and $\mathbf{R}_{y y}=E\left[\mathbf{y}(n) \mathbf{y}^{\dagger}(n)\right]$. Then, $\mathbf{R}_{y y}=\mathbf{T R}_{x x} \mathbf{T}^{\dagger}$ and we have

$$
\begin{aligned}
\prod_{i=0}^{M-1} \sigma_{y_{i}}^{2} & =\prod_{i=0}^{M-1}\left[\mathbf{R}_{y y}\right]_{i i} \geq \operatorname{det} \mathbf{R}_{y y} \\
& =\left[\operatorname{det} \mathbf{R}_{x x}\right]|\operatorname{det} \mathbf{T}|^{2}=\left[\operatorname{det} \mathbf{R}_{x x}\right] /|\operatorname{det} \mathbf{S}|^{2} .
\end{aligned}
$$

Combining the preceding two equations, we see that

$$
\begin{gathered}
\mathcal{E} \geq c M 2^{-2 b}\left(\operatorname{det} \mathbf{R}_{x x}\right)^{1 / M}\left(\frac{\prod_{i=0}^{M-1}\left\|\mathbf{s}_{i}\right\|^{2}}{|\operatorname{det} \mathbf{S}|^{2}}\right)^{1 / M} \\
\geq c M 2^{-2 b}\left(\operatorname{det} \mathbf{R}_{x x}\right)^{1 / M}
\end{gathered}
$$

where the last inequality follows from Hadamard's inequality [25], i.e., the product of the norms of the columns $\mathbf{s}_{k}$ of any nonsingular matrix $\mathbf{S}$ is at least as large as the magnitude of the determinant, with equality if and only if the columns are orthogonal, i.e., $\mathbf{S}^{\dagger} \mathbf{S}$ is diagonal. The right-hand side in the preceding equation is precisely the error bound achieved by the optimal unitary transform $\mathbf{T}$ under optimal bit allocation (see [3] or [8, App. C]). Therefore, we cannot do better by making $\mathbf{T}$ nonunitary. Thus, the minimum $\mathcal{E}$ is achieved if and only if $\mathbf{S}^{\dagger} \mathbf{S}$ is diagonal, i.e., $\mathbf{T T}^{\dagger}$ is diagonal. By normalizing the rows of $\mathbf{T}$ (which does not affect the reconstruction error), we can take $\mathbf{T}$ to be unitary.

\section{REFERENCES}

[1] Y. Huang and P. M. Schultheiss, "Block quantization of correlated Gaussian random variables," IEEE Trans. Commun. Syst., vol. C-10, pp. 289-296, Sept. 1963.

[2] A. Segall, "Bit allocation and encoding for vector sources," IEEE Trans. Inform. Theory, vol. IT-22, pp. 162-169, Mar. 1976.

[3] N. S. Jayant and P. Noll, Digital Coding of Waveforms. Englewood Cliffs, NJ: Prentice-Hall, 1984.

[4] A. K. Jain, Fundamentals of Digital Image Processing. Englewood Cliffs, NJ: Prentice-Hall, 1989.

[5] J. W. Woods, Subband Image Coding. Boston, MA: Kluwer, 1991.

[6] A. Gersho and R. M. Gray, Vector Quantization and Signal Compression. Boston, MA: Kluwer, 1992.

[7] A. N. Akansu and R. A. Haddad, Multiresolution signal Decomposition: Transforms, Subbands, and Wavelets. New York: Academic, 1992.

[8] P. P. Vaidyanathan, Multirate Systems and Filter Banks. Englewood Cliffs, NJ: Prentice-Hall, 1993.

[9] H. S. Malvar, Signal Processing with Lapped Transforms. Norwood, MA: Artech House, 1992.

[10] M. Vetterli and J. Kovačević, Wavelets and Subband Coding. Englewood Cliffs, NJ: Prentice Hall, 1995

[11] G. Strang and T. Nguyen, Wavelets and Filter Banks. Cambridge, MA: Wellesley-Cambridge, 1996.

[12] A. N. Akansu and Y. Liu, "On signal decomposition techniques," Opt. Eng., vol. 30, July 1991.

[13] R. A. Haddad and K. Park, "Modeling, analysis, and optimum design of quantized $M$-band filter banks," IEEE Trans. Signal Processing, vol. 43, pp. 2540-2549, Nov. 1995.

[14] M. Unser, "On the optimality of ideal filters for pyramid and wavelet signal approximation," IEEE Trans. Signal Processing, vol. 41, pp. 3591-3596, Dec. 1993.

[15] - "An extension of the KLT for wavelets and perfect reconstruction filter banks," in Proc. SPIE 2034, Wavelet Appl. Signal Image Process., San Diego, CA, 1993, pp. 45-56.

[16] M. K. Tsatsanis and G. B. Giannakis, "Principal component filter banks for optimum wavelet analysis," in Proc. 6th Signal Process. Workshop, Stat. Signal Array Process., Oct. 1992, pp. 50-53.
[17] _ _ "Principal component filter banks for optimal multiresolution analysis," IEEE Trans. Signal Processing, vol. 43, pp. 1766-1777, Aug. 1995.

[18] P. Delsarte, B. Macq, and D. Slock, "Signal-adapted multiresolution transform for image coding," IEEE Trans. Inform. Theory, vol. 38, pp. 897-904, Mar. 1992.

[19] S. Ohno and H. Sakai, "Optimization of filter banks using cyclostationary spectral analysis," in Proc. Int. Conf. Acoust., Speech, Signal Process., Detroit, MI, May 1995, pp. 1292-1295.

[20] P. Moulin, "A new look at signal-adapted QMF bank design," in Proc. Int. Conf. Acoust., Speech, Signal Process., Detroit, MI, May 1995, pp. 1312-1315.

[21] B. Xuan and R. H. Bamberger, "MD paraunitary principal component filter banks," preprint.

[22] I. Djokovic and P. P. Vaidyanathan, "On optimal analysis/synthesis filters for coding gain optimization," IEEE Trans. Signal Processing, vol. 44, pp. 1276-1279, May 1996.

[23] P. P. Vaidyanathan, "Theory of optimal orthonormal filter banks," in Proc. IEEE Int. Conf. Acoust. Speech, Signal Process., Atlanta, GA, May 1996.

[24] , "Properties of optimal compaction filters in subband coding," in Proc. IEEE Signal Processing Workshop, Loen, Norway, Sept. 1996.

[25] R. A. Horn and C. R. Johnson, Matrix Analysis. Cambridge, U.K.: Cambridge Univ. Press, 1985.

[26] T. Berger, Rate Distortion Theory. Englewood Cliffs, NJ: Prentice-Hall, 1971.

[27] J. Makhoul, S. Roucos, and H. Gish, "Vector quantization in speech coding," Proc. IEEE, vol. 73, pp. 1551-1588, Nov. 1985

[28] P. P. Vaidyanathan, "Review of recent results on optimal orthonormal subband coders," in SPIE Conf. Wavelet Applications Image Signal Process., July/Aug. 1997.

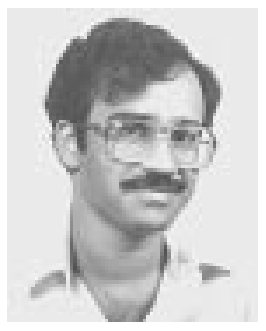

P. P. Vaidyanathan (S'80-M'83-SM'88-F'91) was born in Calcutta, India, on October 16, 1954. He received the B.Sc. (Honors) degree in physics and the B.Tech. and M. Tech. degrees in radiophysics and electronics, all from the University of Calcutta, in 1974, 1977, and 1979, respectively. He received the Ph.D. degree in electrical and computer engineering from the University of California, Santa Barbara, in 1982.

He was a post doctoral Fellow at the University of California, Santa Barbara, from September 1982 to March 1983. In March 1983, he joined the Electrical Engineering Department, California Institute of Technology, Pasadena, as an Assistant Professor, and since 1993, has been a Professor of Electrical Engineering there. His main interests are in digital signal processing, multirate systems, wavelet transforms, and adaptive filtering.

Dr. Vaidyanathan served as Vice Chairman of the Technical Program Commitee for the 1983 IEEE International Symposium on Circuits and Systems and as the Technical Program Chairman for the 1992 IEEE International Symposium on Circuits and Systems. He was an Associate Editor for the IEEE TRansactions on CirCuITS AND Systems from 1985 to 1987 and is currently an Associate Editor for the IEEE SignAL Processing LETTERS and a consulting editor for the journal Applied and Computational Harmonic Analysis. He has authored a number of papers in IEEE journals and is the author of the book Multirate Systems and Filter Banks (Englewood Cliffs, NJ: Prentice-Hall, 1993). He has written several chapters for various signal processing handbooks. He was a recipient of the Award for Excellence in Teaching at the California Institute of Technology during 1983-1984, 1992-1993, and 1993-1994. He also received the NSF's Presidential Young Investigator Award in 1986. In 1989, he received the IEEE ASSP Senior Award for his paper on multirate perfect-reconstruction filter banks. In 1990, he was the recipient of the S. K. Mitra Memorial Award from the Institute of Electronics and Telecommunications Engineers, India, for his joint paper in the IETE journal. He was also the coauthor of a paper on linear-phase perfect reconstruction filter banks in the IEEE TRANSACTIONS ON Signal PROCESSING, for which the first author, T. Nguyen, received the Young Outstanding Author Award in 1993. He received the 1995 F. E. Terman Award of the American Society for Engineering Education, sponsored by Hewlett-Packard Company, for his contributions to engineering education, especially the book, Multirate Systems and Filter Banks. He was named a distinguished lecturer for the IEEE Signal Processing Society during 1996-1997. 